\title{
1 An experimental study of die filling of pharmaceutical powders using a rotary
} die filling system

3 A. Zakhvatayeva ${ }^{1}$, W. Zhong ${ }^{1,2}$, H.A. Makroo ${ }^{1,3}$, C. Hare ${ }^{1}$, C.Y. Wu ${ }^{1, *}$

$4{ }^{1}$ Department of Chemical and Process Engineering, University of Surrey, Guildford, 5 GU2 $7 X H, U K$

$6{ }^{2}$ School of Mechanical Engineering, University of Jinan, Shandong, 250022, China

$7 \quad{ }^{3}$ Department of Food Engineering and Technology, Tezpur University, Napaam

8 Assam, 784028, India

\section{Abstract}

10 Die filling is a critical process step in pharmaceutical tablet manufacturing. Mass and 11 content uniformity of the tablets as well as production throughput depend upon the die 12 filling performance of the formulations. The efficiency of the die filling process is 13 influenced by powder properties, such as flowability, cohesion, particle size and morphology, as well as the process conditions. It is hence important to understand the influence of powder properties on the die filling performance. The purpose of the present study is to identify the critical material attributes that determine the efficiency of die filling. For this purpose, a model rotary die filling system was developed to mimic the die filling process in a typical rotary tablet press. The system consists of a round die table of $500 \mathrm{~mm}$ diameter, equipped with a rectangular die. The die table can rotate at an equivalent translational velocity of up to $1.5 \mathrm{~m} / \mathrm{s}$. The filling occurs when the die passes through a stationary shoe positioned above the die table. Using this system, die filling behaviours of 7 commonly used pharmaceutical excipients with various

\footnotetext{
* Corresponding Author. Tel: +44 1483 683596. Email: C.Y.Wu@surrey.ac.uk
} 
material characteristics (e.g. particle size distribution, sphericity and morphology) and flow properties were examined. The efficiency of die filling is evaluated using the concept of critical filling velocity. It was found that the critical filling velocity is strongly dependent on such properties as cohesion, flowability, average particle size and air sensitivity index. In particular, the critical filling velocity increases proportionally as the mean particle size, flow function, air permeability and air sensitivity index increase, while it decreases with the increase of specific energy and cohesion.

Keywords: Die filling; Tabletting; Flowability; Air sensitivity; Rotary press; Critical filling velocity.

\section{Introduction}

Powder compaction is widely employed in manufacturing of tablets, which are the most common solid dosage forms in the pharmaceutical industry. The same manufacturing process is also widely used in such fields as powder metallurgy, ceramic and chemical industries. During powder compaction, a powder is first deposited into a die, which is the so-called die filling stage, and then compressed under high pressure to form a coherent powder compact.

The most important parameters in die filling are the weight consistency and uniformity, which are heavily influenced by the powder properties, particularly by its flowability. This explains why a significant amount of experimental work and computational studies have been devoted to the powder flow characterization and prediction, as described below. It has been shown that the flow behaviour is significantly influenced by powder characteristics as well as the filling system and the process conditions. 
47 Powder flow from a moving shoe was investigated by Wu et al. [1], who described two types of flow that contribute to the cavity filling: nose and bulk flow. Nose flow, which prevails at low velocities, is the most efficient in filling the die completely. As the shoe accelerates to reach the requested velocity, the powder contained in it moves towards the back of the shoe, forming a nose-shaped profile. The particles on the top of the powder can move more freely than the bottom layers as the shoe starts moving over the die. This kind of flow allows the air trapped in the die to escape more readily, thus improving the die filling efficiency. As the powder bed continues moving over the die, some particles start detaching from the bottom of the powder bed as well. This type of flow is known as bulk flow and it becomes predominant at high filling velocities, at which the shoe completely covers the die during powder discharge. Therefore, the filling velocity (i.e. the velocity of the shoe with respect to the stationary die) is an important factor. Generally, the slower the filling velocity the higher the fill density. However, it was also shown that the fill density can increase as the shoe velocity increases [2], especially when a wide or a large die is used. It was hypothesised that this occurs as the filling process progresses due to the powder stream entering the die at a greater velocity, and so causing compaction as it collides with the powder already present in the die compacting, thus increasing the fill density.

The effects of gravity and vacuum filling on powder filling behaviour were investigated by Sinka et al. [3]. A series of non-dimensional parameters, such as dimensionless mass flow rate and velocity (in vacuum filling), were identified to describe the powder flow mechanism in a die. In the case of gravity filling of cohesive powders in the presence of air, the flow behaviour was described using non-dimensional parameters that take into account the magnitude of the pressure gradient created as the powder falls into the die. Using the data obtained from vacuum filling tests, the maximum 
rotational velocity of a rotary tablet press that would allow the die to be completely filled was predicted. For this purpose, the parameters correlated to powder properties (coefficients of inter-particle sliding friction and sliding friction between the particles and the base plate on which the shoe moves) and the shape of the die opening, obtained from die filling tests in vacuum, were used, as they were deemed appropriate as a first approximation for the conditions in the tablet press. It was shown that the predicted value was approximately half of the tablet press velocity normally used in manufacturing. The authors then argued that the considered parameters, determined from vacuum filling tests, may underestimate the enhanced effect of the suction filling mechanism in a tablet press. The importance of suction on the die filling efficiency was demonstrated by Jackson et al. [4]. The authors used a modified linear die filling system, equipped with a suction piston, to investigate the effect of suction on the filling efficiency of microcrystalline cellulose. Using this result, Sinka et al. [3] were able to predict a maximum rotational velocity that was consistent with values used normally in the tablet manufacturing. It was concluded that further studies are necessary for the up-scaling of the suction filling kinematics, including the effect of using a paddle feeder (forced feeding), in order to extrapolate more reliable results from experimental die filling to real tablet presses.

Powder flow behaviour during die filling with dies of different size and shape both in vacuum and in the presence of air was examined by Wu et al. [1][5]. The concept of critical filling velocity, above which the die is only partially filled, was also introduced to characterise powder flowability with specific application to die filling. The efficiency of die filling was expressed in terms of the variation of the fill ratio, $\delta$, with the filling velocity. The fill ratio was defined as: 
where $m$ is the mass of powder in the die at a given fill velocity and $M$ is the mass of powder in a fully filled die. For filling velocities above the critical velocity, the fill ratio is below 1.0, and the fill ratio can be expressed as a function of the filling velocity as follows:

$$
\delta=\left(\frac{v_{c}}{v}\right)^{n} \quad\left(v>v_{c}\right)
$$

where $v_{c}$ is the critical velocity, $v$ is the filling velocity $\left(v>v_{c}\right)$ and $n$ is a materialdependent parameter, which was found to have values between 0.9 and 1.6 for the powders tested [5]. For filling velocities lower than $v_{c}$ the fill ratio, $\delta$, is equal to 1.

The concept of critical velocity was also suggested for measuring powder flowability with special applications to die filling [6]. It was also suggested that the use of a testing device that mimics the actual conditions of a rotary tablet would be preferable to assess the powder flowability in relation to the die filling process.

The influence of powder properties on the die filling behaviour was also evaluated. For instance, Mills et al. [7] investigated the die filling behaviour of various grades of microcrystalline cellulose using a linear die filing system. A strong relationship between the critical filling velocity and the particle size was demonstrated. It was shown that powders with finer particles generally exhibit higher cohesion and intermittent die filling behaviour. In addition, the critical filling velocity can be significantly increased with suction filling. However, the increase in fill ratio with addition of suction pressure causes a more pronounced increase in fill ratio for smaller 
particles, since larger particles (generally having better air permeability) allow air to escape more easily and thus are less affected by the suction.

The influence of powder properties on the die filling was investigated by Freeman et al. [8], using a linear filling rig similar to the one used by Wu et al. [1]. It was concluded that the die filling efficiency is increased for powders with low values of basic flow energy (BFE), flowrate index (assessed through Stability and Variable Flow Rate tests), consolidation index (ratio between flow energy after consolidation and BFE), shear strength, cohesion, internal angle of friction (assessed with the shear cell test) and compressibility, which represents the variation of powder volume as it is gradually compressed under increasing value of normal stress.

The die filling efficiency is influenced by the presence of air, especially for air-sensitive powders. According to Guo et al. [9], the sensitivity of particles to air can be expressed using a dimensionless parameter:

$$
\xi=A_{r} \Phi_{\rho}
$$

where $\Phi_{\rho}$ (normalised particle density) is the ratio of the solid true density $\left(\rho_{s}\right)$ to the air density $\left(\rho_{a}\right)$, and $\mathrm{A}_{\mathrm{r}}$ is the Archimedes number of particles flowing in air, given by:

$$
A_{r}=\frac{\rho_{a}\left(\rho_{s}-\rho_{a}\right) g d_{p}^{3}}{\eta^{2}}
$$

where $d_{p}$ is the particle diameter, $\eta$ is the air viscosity and $g$ is gravitational acceleration. It was shown that the higher the value of $\xi$ the lower the effect of air on the fill ratio. The dimensionless parameter $\xi$ can be used to classify the powder as airsensitive or air-inert, depending on the regime of flow behaviour during die filling in air. For $\xi$ values lower than a critical value of $9.6 \times 10^{6}$, the powder flow rate is heavily hindered by the presence of air, which is the typical behaviour of small and light 
141 particles. This regime is referred to as air-sensitive. Contrarily, powders with a value of $\xi$ higher than the critical value, represented by heavy and large particles, belong to the air-inert regime.

Several studies were also performed for rotary die filling; where the shoe is stationary and the die is attached to a rotating die table, allowing it to pass below the shoe, and thus more closely representing tablet presses. The effects of the paddle speed on the powder flow behaviour and the filling uniformity using a model rotary die table fitted with a standard feed frame were investigated by Mendez et al. [10]. The examined powders were characterised in terms of the flow index using a gravitational displacement rheometer (GDR), which evaluates the change in moment of inertia of a powder loaded into a rotating drum [11]. The importance of the feeding speed and frame geometry as well as the powder flowability on the uniformity and efficiency of die filling were explored. It was shown that the efficiency of die filling increases as the feed frame speed increases and as the die table speed decreases. Higher values of feed frame speed on one hand also reduced the weight variation, but on the other maximized the tendency to overlubricate. The authors argued the necessity to address the issue in future studies. Further studies on the influence of powder properties on the die filling behaviour in tabletting were carried out by Peeters et al. [12] who investigated the flow behaviour of dibasic calcium phosphate dihydrate (DCPD) and microcrystalline cellulose (MCC) Avicel PH102. The powders were characterized in terms of particle size distribution, density and compressibility index. The tablet weight variability and the volume of the powder inside the feeder were assessed as a function of fill depth, tableting speed and paddle speed. It was shown that weight uniformity was influenced by the paddle speed, particularly for cohesive powders. The tabletting speed had a negative effect on the uniformity. The overall variability for MCC tablets 
was higher than that for DCP tablets, which was attributed to the difference in powder flowability: based on the compressibility index, MCC was described as a fairly flowing material, whereas DCP had excellent flow properties.

In a recent study, Van Snick et al. [13] investigated the influence of powder properties and process parameters on the product and process responses. Binary blends consisting of an active ingredient and a filler were considered using 6 different APIs and 5 fillers. The blends were characterised in terms of compressibility, density, permeability, wall friction and porosity. The blends were tableted on a rotary tablet press, varying the paddle and the turret speed according to a two-level full factorial 
identify a single flowability indicator suitable for die filling that takes into account the most significant powder characteristics. The concept of critical velocity introduced by Wu et al. [1][6] was demonstrated suitable for describing the flow performance in the linear die filling [8], but it is still not clear if this concept is suitable for rotary die filling. Therefore, the aim of this study is to explore if the die filling behaviour of powders during rotary die filling can be assessed using the critical filling speeds and if there is any correlation between the critical filling speed with powder properties. For this purpose, a rotary die filling system was developed to mimic the actual filling process in a rotary press, where the feeder is stationary and the filling is carried out by feeding the powder in a die, positioned on a rotating die table. In addition, a systematic investigation with a wide range of pharmaceutical powders is performed.

\section{Materials and methods}

The powders selected in this study are commonly used pharmaceutical excipients, including microcrystalline cellulose of two different grades: MCC Avicel PH101 and PH102 (FMC; Biopolymer, Cork, Ireland), co-processed microcrystalline cellulose and anhydrous dibasic calcium phosphate, referred to as MCC DG (FMC Biopolymer, Cork, Ireland), microcrystalline cellulose spheres, referred to as MCC CP102 (CELPHERE ${ }^{\circledR}$ CP102; Asahi Kasei, Japan), lactose monohydrate (Granulac ${ }^{\circledR} 140$; Meggle, Germany), mannitol (Pearlitol ${ }^{\circledR}$ 100SD; Roquette Pharma, France) and calcium phosphate dibasic anhydrous, referred to as DCPA (Emcompress ${ }^{\circledR}$; JRS PHARMA GMBH \& Co., Rosenberg, Germany). 


\subsection{Particle size and morphology analysis}

215

216

217

218

219

220

221

222

The QICPIC particle size analyser equipped with the GRADIS dry powder disperser (Sympatec, Inc., Clausthal-Zellerfeld, Germany) was used to measure particle size and sphericity distributions. The measure of particle size used is defined in terms of equivalent projection circle (EQPC), which is the diameter of a circle that has the same area as the projected area of the particle. The samples were fed through a gap of 4 $\mathrm{mm}$ at a feed rate of $10 \%$ of the maximum feed rate of a VIBRI vibratory feeder. Each sample, collected from different points in the bulk material, was analysed three times. The size parameters $d_{10}, d_{50}, d_{90}$, span $(\psi)$ as well as sphericity cumulative distribution $Q_{3}$, shape parameters $s_{10}, s_{50}$ and $s_{90}$ were determined. The span a measure of the distribution width, is calculated as follows:

$$
\psi=\frac{d_{90}-d_{10}}{d_{50}}
$$

Sphericity is defined as the ratio of the perimeter of the circle with the same projected area as the particle to the measured perimeter of the projected particle. The cumulative sphericity distribution is the fraction of particles (based on volume) with sphericity less than a certain value.

The shape and surface features of the powder particles are assessed using scanning electron microscopy (SEM). The samples were prepared using colloidal graphite in order to immobilize the particles on the sample holder and are subsequently goldcoated. The observations are carried out using a Jeol $7100 \mathrm{~F}$ thermal field emission electron microscope (Jeol Ltd., Tokyo, Japan) with $3 \mathrm{kV}$ accelerating voltage at different magnifications. 


\subsection{Flow index and mass flow rate}

238

239

240

241

242

243

244

245

246

247

248

249

250

251

252

253

254

The Flodex ${ }^{\circledR}$ flowmeter (Hanson Research Corporation, Chatsworth, USA) was used to assess the minimum orifice diameter through which the powder could flow freely. The device consists of a stainless steel cylinder, equipped with interchangeable disks with orifices of diameter ranging from 4 to $34 \mathrm{~mm}$. The cylinder was filled with a tested powder through a funnel. The amount of the tested powder was usually around $50 \mathrm{~g}$, but the amount of powder used is volume-controlled, i.e. adjusted to fill the cylinder to within $1 \mathrm{~cm}$ from the top. The release lever under the cylinder was slowly turned to open the disc and allow powder to flow through the orifice. If the hole at the bottom of the cylinder was visible after powder discharge, it indicated that the powder could flow through the considered orifice. The test was repeated by decreasing the orifice diameter until no flow was observed. The diameter of the smallest orifice that enabled the powder flow was used as the flow index.

Once the least free-flowing material (which required the largest opening for it to flow) was identified using the Flodex, the corresponding disc was used to measure the mass flow rate. The same amount of material as used for the flow index test was used to fill the cylinder. All the powders were made to flow through the same disc into a container that was positioned on a high precision miniature load cell HBM PW4M (HBM Ltd., North Harrow, UK). The load cell was connected to a data logger (PICO high resolution data logger ADC-20, PICO Technology Ltd., United Kingdom) that converted the voltage to mass, so that the mass of the discharged material could be measured during the discharge process.

The fraction of discharged mass at each time instant then could be determined as:

$$
f=\frac{m_{f}}{M_{f}}
$$


261 where $m_{f}$ is the mass of powder that flows through the orifice and $M_{f}$ is the total amount

262 of powder loaded in the cylinder.

263 The mass flowrate is then defined as follows:

$$
\bar{m}=\frac{\Delta m_{f}}{\Delta \mathrm{t}}
$$

where $\Delta m_{f}$ is the discharged mass and $\Delta \mathrm{t}$ is the discharge time.

\subsection{Flow properties}

An FT4 powder rheometer (Freeman Technology, Tewkesbury, UK) was used to assess the flow properties such as specific energy (SE), air permeability, conditioned bulk density, flow function and cohesion.

The evaluation of specific energy consists of a dynamic test that measures the resistance of particles moving relative to each other in an unconfined state. For this purpose, a vessel of $50 \mathrm{~mm}$ diameter and a twisted blade of $48 \mathrm{~mm}$ diameter were used. The vessel was filled with the powder to a bed height greater than $80 \mathrm{~mm}$, conditioned by the blade, and then the excess powder beyond $80 \mathrm{~mm}$ height was removed with the split cell. The SE value is equal to the work done by the blade while moving through the analysed powder from a height of $10 \mathrm{~mm}$ from the base up to the top of vessel, divided by the total powder mass. SE gives an indication of the degree of particle interlocking that limits the powder flow. The lower the SE value, the lower the mechanical interlocking and the more likely the powder is to flow under gravity in an unconfined state [14][15][16]. The air permeability test measures how easily air can pass through a powder bed. Low values of permeability (and therefore high values of pressure drop across the test container) result in intermittent flow and compromised 
filling. The permeability is measured in terms of pressure drop across the powder bed, contained in a $50 \mathrm{~mm}$ vessel, while air is introduced to the bottom of the test vessel at a constant velocity $(2 \mathrm{~mm} / \mathrm{s})$. The powder is also subjected to mild consolidation under a range of stresses by means of a vented piston, equipped with fine mesh that allows measured at the bottom of the vessel represents the resistance of the powder to air flow at a given level of consolidation and air velocity.

Flow function and cohesion were obtained from the rotational shear test. For this purpose, a shear cell module of the FT4 was used. The evaluation of shear properties was carried out using a cylindrical vessel of $50 \mathrm{~mm}$ diameter and a volume of $85 \mathrm{~cm}^{3}$.

The bed was pre-consolidated using the standard shear cell test with the minimum normal stress of $3 \mathrm{kPa}$ and pre-sheared until a steady state shear stress was established. A lower normal stress was applied and the peak shear stress at incipient

Both the flow function and cohesion depend on the material properties and can be 


\subsection{Air sensitivity index}

309

310

312

313

314

The values of air sensitivity index for all powders were calculated according to Equation (3):

$$
\xi=A_{r} \Phi_{\rho}
$$

where $\Phi_{\rho}$, the normalised particle density, is calculated as the ratio of the solid true density, $\rho_{\mathrm{s}}$, to the air density $\rho_{\mathrm{a}}\left(1.205 \mathrm{~kg} / \mathrm{m}^{3}\right)$. The Archimedes number of particles flowing in air, $A_{r}$, is given by:

$$
A_{r}=\frac{\rho_{a}\left(\rho_{s}-\rho_{a}\right) g d_{p}^{3}}{\eta^{2}}
$$

where $d_{p}$ is the particle diameter $\left(d_{50}\right), \eta$ is the air viscosity $\left(1.78^{*} 10^{-5} \mathrm{Ns} / \mathrm{m}^{2}\right)$ and $g$ is gravitational acceleration.

\subsection{Die filling}

A rotary die filling system was developed to explore the die filling behaviour (Figure 1). The rotary die filling system consists of a circular die table with pitch circle radius (distance from the centre of the table to the centre of the die) of $246 \mathrm{~mm}$. The die table is equipped with a transparent rectangular-shaped die with a cross section of $25 \mathrm{~mm}$ $x 25 \mathrm{~mm}$ and a depth of $60 \mathrm{~mm}$. A stationary feeder is positioned above but in close contact with the die table. The powder is manually poured into the feeder with excess powder being levelled off so that the same volume of powder is used for each test. The feeder shape is curved so that the geometrical centre of the die during its transit under the shoe is kept central to a filling area of $30 \mathrm{~mm}$ width and covers an angle of $45^{\circ}$. The powder is fed by gravity as the feeder is not equipped with forced feeding elements (paddles). The die table velocity is set in the range of $5-550 \mathrm{~mm} / \mathrm{s}$ and each 
test is run in triplicate. The die filling efficiency is evaluated in terms of the fill ratio as a function of the filling velocity, as defined by Equation (1), where $m$, the mass of powder in the die at a given fill velocity, is evaluated by weighing the die before and after the filling step, and $M$, the mass of powder in a fully filled die, is evaluated by weighing the die before and after the filling step at a low velocity, at which the die is completely filled. The dynamics of the filling process are also captured with a high speed camera at $1000 \mathrm{fps}$ (Phantom VEO, Vision Research Inc., USA).

\section{Results and discussion}

\subsection{Particle size and morphology}

Particle size and shape distributions, and morphology of the seven powders considered are presented in Figures 2 - 4, respectively, and characteristic size and shape parameters are given in Table 1. It can be seen that lactose monohydrate, MCC DG and MCC PH101 are very similar in terms of $d_{50}$ and of the distribution width, while DCPA, MCC CP102 and mannitol form a group with coarser particles and narrower distributions. MCC PH102 is characterised by an intermediate position in terms of particle size, whereas the span is the same as the three fine powders.

In terms of sphericity (Figure 3), the powder ranking is quite similar to that of particle size, with the exception of lactose monohydrate, which shows quite high values of sphericity, comparable to that of mannitol. As can be seen from the SEM images (Figure 4a), lactose particles appear more spherical. The SEM images also confirm a higher degree of sphericity for coarser particles of Mannitol, DCPA and MCC CP102 (Figures $4 e, 4 f, 4 g$ ). DCPA's crystalline structure is very evident (see Figure $4 f)$. The 
particles of Lactose, MCC DG, MCC PH101 and MCC PH102 (Figures 4a-4d, respectively) also have more irregular shape and notable surface roughness.

\subsection{Mass flow rate}

The variation of discharged mass fraction, calculated according to Equaton (6), as a function of time (evaluated with Flode ${ }^{\circledR}$ ) for the seven powders is presented in Figure 5. The discharge of material though the orifice is not constant in time for lactose, MCC DG and MCC PH101. The discharge occurs intermittently: when the shutter is opened, a lump of material falls through the orifice under the effect of its own weight. In the case of lactose, this happens in the first $100 \mathrm{~ms}$. After this phase, the slope of the linear part of the curve decreases and then a second, smaller portion of material falls from the walls of the channel (surrounding the 'rathole') created by the first discharge. The plateau value represents the final mass fraction that is the ratio between the amount of powder discharged at the end of the test and the total amount of powder loaded in the cylinder. It can be seen, that for lactose the mass fraction at the end of the test is very low (around 0.30 ), while it is much higher for the other materials. The discharge of MCC PH101 is less intermittent than lactose, and the final mass fraction is around 0.50 . For MCC DG, the final mass fraction is also around 0.50 , but it is reached in a shorter time than for lactose and MCC DG. For MCC PH102 the discharge occurs more slowly but the final mass fraction is higher than that for lactose, MCC DG and MCC PH101. The mass fraction curve of mannitol has a more constant slope, with a higher value of final mass fraction if compared to MCC PH102. Finally, for both DCPA and MCC CP102 the slope of the curve is quite constant and the final 
mass fraction values are the highest considered (around 0.80 for DCPA and 0.85 for MCC CP102) among the materials.

The behaviour observed above is a direct consequence of the powder properties. The intermittent flow of lactose, MCC DG and MCC PH101 is attributed to their small particle size and irregular shape (Figure $4 a$, Figure $4 b$, Figure $4 c$ ), which cause particles to interlock and flow in lumps. For MCC PH102, the interparticle cohesion is relatively low (larger particles and smoother surfaces, see Figure $4 d$ ) therefore the flow does not occur intermittently but in a more continuous and smooth manner, similar to that of the free flowing materials. Unlike the free flowing materials though, MCC PH102 still has sufficient cohesion to inhibit the free flow of the bulk mass. Mannitol exhibits a more stable flow due to its higher sphericity, but the roughness and a relatively low value of $d_{50}$ still cause the final mass fraction to be only slightly higher than that of MCC PH102.The other two powders (DCPA and MCC CP102) exhibit greater and more consistent mass flow rates, due to their highly spherical and larger particles.

Table 2 presents the values of mass flow rate, calculated according to Equation (7). It is clear that the lowest values of mass flow rate are observed for the three finest powders (lactose, MCC DG and MCC PH101) and for MCC PH102. The highest values of mass flow rate are obtained for the free flowing materials (MCC CP102, Mannitol and DCPA).

In the last column of Table 2, the values of flow index and mass flow rate through a 24 $\mathrm{mm}$ diameter orifice are presented. From the flow index ranking it can be seen that the powders with the highest flow index values are lactose and MCC DG, which also have the lowest values of $d_{50}$ (Table 1). Generally, the flow index decreases as $d_{50}$ 
401

402

403

404

405

406

407

408

409

410

411

412

413

increases; MCC PH101, with slightly coarser particles than MCC DG, has a flow index value of 20 whereas MCC DG has a flow index of 22 . The materials with the highest values of $d_{50}$ are mannitol, DCPA and MCC CP102, and they also have the lowest values of flow index. Finally, the particle size and flow index of MCC PH102 lie in between those two groups of powders.

\subsection{Flow properties}

The corresponding pressure drop values as a function of the applied normal stress are shown in Figure 6. It appears that the pressure drop is not sensitive to the applied normal stress for most powders considered, while for lactose, the pressure drop is not only the highest at a given applied normal stress, but also increases significantly with the applied normal stress. It is evident that the highest pressure drop is observed for lactose, MCC DG and MCC PH101, whereas the lowest pressure drop is observed in the case of MCC PH102, mannitol, DCPA and MCC CP102. The highest pressure drop for lactose may be due to a series of factors such as small particle size, irregular shape and a wide particle size distribution (that gives rise to a tightly packed structure). It has also been confirmed by Hertel et al. through studies on dry powder inhaler formulations that for lactose a high fines content reduces the permeability value at a given applied normal pressure [17]. The measured values of specific energy and pressure drop at maximum normal stress of $15 \mathrm{kPa}$ are presented in Table 3 . The specific energy is greatest lactose, MCC DG, MCC PH101 and MCC PH102, and the lowest for mannitol, DCPA and MCC CP102. This is believed to be due to the degree of particle interlocking [14]. The powders with the most irregularly shaped particles (observed from SEM, see Fig.2), and therefore the highest degree of interlocking, are lactose, MCC DG, MCC PH101 and MCC PH102 which are also the finest and least free flowing (Table 1 and Table 2). Based on specific energy, the three most free 
426 flowing materials are DCPA, Mannitol and MCC CP102, due to the more regular

427 particle shape and larger size.

428 The flow function and cohesion values obtained from the shear cell tests are presented

429 in Table 4. The lowest values of flow function (ffc) are observed for the least free 430 flowing powders, lactose, MCC DG and MCC PH101. The highest flow function values 431 are exhibited by DCPA and mannitol, which can be described as free flowing. The flow 432 function value measured for MCC CP102 appears to be lower than expected. It is to 433 be considered that MCC CP102 consists of microspheres of almost perfectly spherical 434 shape and therefore the variability in the shear behaviour of MCC CP102 is higher 435 than for the other, non-spherical, materials. For MCC PH102 the flow function value 436 lies between the two considered groups (with the exception of MCC CP102). As for 437 the cohesion, the highest values are observed for lactose, MCC DG and MCC PH101 438 (which have the lowest flow function values), whereas the lowest values are exhibited 439 by MCC PH102, mannitol, DCPA and MCC CP102..

\subsection{Air sensitivity index}

442 The values of air-sensitivity index are presented in Table 5. It can be seen that the airsensitivity index for all the samples considered are below the critical value. Therefore, for all of them the filling process is expected to be influenced by the presence of air. Figure 7 presents the distribution of air sensitivity values and the predicted dimensionless mass flow rate using the formula given by Guo et al. [8], i.e.:

$$
\mathrm{M}^{*}=0.233\left(\mathrm{~A}_{\mathrm{r}} \Phi\right)^{\frac{1}{5}}
$$

It can be seen that lactose, MCC DG and MCC PH101 have the lowest air-sensitivity index and are hence the most air-sensitive materials, followed by MCC PH102 and 
mannitol, MCC CP102 and DCPA. The highest value of air-sensitivity index exhibited by DCPA is due to its high true density and particle size.

\subsection{Die filling performance}

The flow profiles of different powders are shown in the high speed camera snapshots of Figure 8 . The sequence of Figure $8 a$ shows the die filling profile of lactose monohydrate at two different filling velocities: the low velocity, at which the die is completely filled, and the high velocity at which it is only partially filled. It can be seen at low velocities that the flow of lactose occurs intermittently, i.e. lumps of powder detach from the shoe and fall into the die [18]. At higher filling velocities the flow profile remains intermittent and the die filling is incomplete, as the filling velocity is higher than the critical filling velocity [5]. Die filling of MCC DG (Figure 8b) also occurs intermittently, although the lumps of material are smaller and the overall flow profile is smoother than that of lactose. A similar flow profile is observed for MCC PH101 (Figure $8 c$ ). The filling still occurs with detachment of lumps of powder, although they are smaller than for lactose and MCC DG.

For MCC PH102 (Figure 8d) the flow becomes smooth and continuous, as was similarly observed by Sinka et al. in linear die filling in presence of air [18]. The flow is continuous as well in case of mannitol (Figure 8e), DCPA (Figure 8f) and MCC CP102 (Figure 8g).

The efficiency of die filling is graphically presented in Figure 9, in which the fill ratio is plotted against the filling velocity. For clarity, Figure 9a presents the fill ratio as a function of filling velocity for lactose, MCC PH101, MCC DG and MCC PH102. It can be seen that for these powders the die is completely full (fill ratio $\cong 1.0$ ) at filling 
474 velocities significantly below $100 \mathrm{~mm} / \mathrm{s}$ (for lactose this velocity is closer to $10 \mathrm{~mm} / \mathrm{s}$ ).

475 The fill ratio then decreases exponentially and abruptly as the filling velocity increases. For lactose and MCC DG the fill ratio approaches $0.10-0.15$ at $100-150 \mathrm{~mm} / \mathrm{s}$, whereas for MCC PH101 and PH102 a filling velocity of $400 \mathrm{~mm} / \mathrm{s}$ is necessary to reduce the fill ratio to $0.15-0.20$. As for the more freely flowing materials in Figure $9 b$, the die is still completely full at higher velocities $(100-150 \mathrm{~mm} / \mathrm{s})$ and the fill ratio decreases less sharply as the filling velocity increases. The standard error associated with each data point is generally smaller than that of the more cohesive powders, like lactose, MCC DG and MCC PH101. The flow of mannitol. DCPA and MCC CP102 is therefore more stable and reproducible.

The data presented in Fig. 9 were then used to calculate the critical filling velocity $\left(v_{c}\right)$ and the material-dependent exponent $n$, according to Equation (2). The resulting values are presented in Table 6. It can be seen that lactose, MCC DG and MCC PH101 show the lowest values of critical filling velocity, which means that the die is completely filled at comparatively low values of filling velocity. This is due to high cohesion, low particle size and high air sensitivity. The higher cohesion values (Table 4) and higher particle interlocking due to higher surface area (smaller particles) interfere with the powder flow in the die and particles tend to detach in larger aggregates rather than in a continuous stream. These powders are also very air-sensitive, meaning that the flow of powder in the die is inhibited by the entrapped air that tries to escape from the die during the filling step [5]. Mannitol, DCPA and MCC CP102, on the other hand, present the highest values of critical filling velocity, i.e. they can fill the die completely at significantly higher values of filling velocity. Specifically, mannitol has the lowest critical

497 filling velocity among the free flowing materials (DCPA and MCC CP102), which could be caused by its comparatively smaller particle size and higher air sensitivity. On the 
other hand, the critical filling velocity of DCPA is higher since the particles are coarser and the true density value is significantly higher than that of mannitol (Table 5). Finally, the highest critical filling velocity of MCC CP102 is attributed to both its large particle size and high sphericity, which result in free flowing behaviour. It could be expected that the true density and particle size of MCC CP102 should lead to a lower critical filling velocity than DCPA (due to the lower $A_{r}$ value), but evidently in the case of die filling the shape factor plays an important role. Finally, MCC PH102 is again positioned between the two groups, one consisting of lactose, MCC DG, MCC PH101 and the other consisting of mannitol, DCPA and MCC CP102. This can be expected, since it has intermediate values in terms of particle size, specific energy, flow function and air sensitivity.

It can be seen that the considered materials can be grouped in terms of average particle size, mass flow rate, specific energy, flow function, cohesion, air sensitivity index and critical velocity, as follows:

group 1: comprises lactose monohydrate, MCC DG and MCC PH101, characterized by $d_{50}$ between $80-90 \mu \mathrm{m}$ and large span (Table 1 ), lower values of mass flow rate $(47.4-60 \mathrm{~g} / \mathrm{s}$, Table 2$)$, higher specific energy $(>8.0 \mathrm{~mJ} / \mathrm{g}$, Table 3), lower flow function $(<15$, Table 4$)$, higher cohesion $(>0.1 \mathrm{kPa}$, Table 4), lower air sensitivity index, and therefore higher air sensitivity $\left(<10^{5}\right.$, Table 5) and lower critical velocity $(<50 \mathrm{~mm} / \mathrm{s}$, Table 6).

group 2: comprises mannitol, DCPA and MCC CP102, characterized by larger particle size ( $d_{50}$ above $\left.120 \mu \mathrm{m}\right)$ and narrow span, higher values of mass flow rate, lower specific energy, higher flow function, lower cohesion, lower air sensitivity and higher critical velocity. 
MCC PH102 is assiged an intermediate position between groups 1 and 2, as It appears in some cases to be closely related to either group 1 (air sensitivity, critical velocity) or 2 (flow function, cohesion).

\subsection{The influence of powder properties on powder flow behaviour}

527 In order to explore how powder flow behaviour depends on the powder properties, especially for rotary die filling, the correlation between the flow index (characterising the powder flow in hoppers) as well as the critical filling velocity (characterising the powder flow performance during die filling) and powder properties (such as particle size, cohesion, air permeability, air sensitivity etc.) is further explored and presented in this section.

\subsubsection{Effect of particle size}

534 The relationship between the flow index and the particle size parameter $d_{50}$ is presented in Figure 10. It is evident that the flow index decreases (and therefore the flowability increases) with the increase of the particle size. It is interesting to note that the flow index decreases sharply with increasing particle size when $d_{50}$ is smaller than $120 \mu \mathrm{m}$ (group 1 powders and MCC PH102), whilst it becomes insensitive to the particle size when $d_{50}$ is larger than $120 \mu \mathrm{m}$ (group 2). This is consistent with the results obtained by Schiano, Chen and Wu [19], which showed that for sufficiently index variation becomes negligible with the particle size increase. This also implies that when particles are large enough and the powder is free flowing, their flow performance cannot be effectively distinguished using the flow index.

Figure 11 shows the correlation between the critical filling velocity and $d_{50}$. It can be seen that the critical filling velocity increases linearly with the increase of $d_{50}$. A linear 
increase of critical velocity with particle size was observed by Schiano et al. [19] who also noticed that eventually the values of critical velocity necessarily reaches a plateau, if the particle size becomes large enough $(>1000 \mu \mathrm{m})$. In the range of $80-200$

$550 \mu \mathrm{m}$ considered in this study the linear trend is evident, even though the most free 551 flowing materials, DCPA and MCC CP102, deviate slightly from the trendline. For MCC 552 CP102 a higher than expected critical filling velocity might be due to its highly spherical particle shape, that improves the die filling behaviour. For DCPA, which consists of coarser particles, the expected critical filling velocity is below the trendline. This could be caused by lower sphericity (Table 1) and more irregular surface of its particles (Figure 4f), compared to MCC CP102 (Figure 4g).

\subsubsection{Effect of specific energy}

559 In Figure 12 the critical filling velocity is plotted against the average values of specific energy. The critical filling velocity generally decreases with the increase of specific energy, as observed by Freeman et al. [8] in their investigation of how glass beads, tungsten powder and aluminium powder granules properties influence the efficiency of die filling in a linear system. Since specific energy is potentially correlated with particle interlocking [14], it is to be expected that powders with the highest specific energy are those that also show low values of critical velocity. The relationship is approximately linear, though some deviation from this trend is noticeable. MCC DG exhibits high variability in specific energy values. The specific energy considers the flow resistance of a powder in an unconfined, low stress state [15][16]. Its value can be therefore influenced by powder packing as the test blade moves through the 
powder bed. For irregular shape particles like MCC DG and lactose, this could lead to variable packing states, which could explain to some extent the observed variation.

\subsubsection{Effect of air permeability}

Figure 13 shows the critical velocity as a function of pressure drop measured at 15 $\mathrm{kPa}$ of applied normal pressure (last data point in Figure 6). It can be seen that an increase in pressure drop measured at $15 \mathrm{kPa}$ leads to a sharp reduction in critical filling velocity up to around 2 mbar (group 2 and MCC PH102), beyond which the change in critical filling velocity is negligible (Group 1). This suggests that pressure drop is not a reliable parameter for predicting critical filling velocity. The value of pressure drop is influenced by the amount of fines [16]: fine powders can form a more compacted bed that cannot easily be permeated by air. Therefore, the highest value of pressure drop is observed for lactose (which contains most fines), which also has the lowest critical filling velocity.

\subsubsection{Effects of flow function and cohesion}

The relationship between the critical filling velocity and the flow function is presented in Figure 14. The critical filling velocity increases linearly with flow function. Freeman et al. [8] observed a similar behaviour investigating flow properties of glass beads, tungsten and aluminium, where values of flow function, calculate at $3 \mathrm{kPa}$ normal stress, appeared to increase with the critical velocity, assessed on a linear die filling rig, similar to one used by Wu [1] and Schneider [3]. A deviation is observed for MCC CP102, which appears to have a higher critical filling velocity than expected, based on its flow function. This could be explained by the fact that at the consolidation stress 
is higher than in case of non-spherical materials. This could be caused by the fact that the precision of flow function measurement can be compromised in case of powders with low cohesion values, as demonstrated by the non-linear mathematical model of Leung et al. [20].

599 The relationship between the critical filling velocity and cohesion is presented in Figure

15. It can be seen that the critical filling velocity decreases sharply with the increasing cohesion when cohesion is lower than approximately $0.15 \mathrm{kPa}$ (group 2 and MCC $\mathrm{PH} 102)$. The critical filling velocity becomes insensitive to the cohesion value when it is higher than $0.15 \mathrm{kPa}$ (group 1). This implies that the cohesion value, unlike the flow function, cannot be used to evaluate the critical filling velocity.

Overall, the relationship between the critical filling velocity and flow function appears to be linear, whereas between the critical velocity and cohesion there seems to be a power relation. It is interesting to note, that the relationship between flow function and cohesion was observed to be an inverse power relation, as shown by Wang et al. [21]. It appears that flow function is more appropriate for critical velocity estimation than cohesion.

\subsubsection{Air sensitivity index}

612 Figure 16 shows the correlation between the critical filling velocity and the logarithm 613 of air sensitivity index. A very clear linear trend can be observed from this figure. DCPA 614 appears as an outlier due to its higher true density (and lower air sensitivity) compared 615 to other investigated materials (Table 6). 


\section{Conclusions}

618 Die filling behaviours of 7 commonly used pharmaceutical excipients were analysed 619 in terms of critical filling velocity, assessed using a rotary die filling system. The 620 calculated values of critical velocity were analysed in terms of their relationship to some relevant powder properties. The results show a good correlation between critical filling velocity and some of the investigated powder properties. Specifically, the critical filling velocity depends strongly on the particle size: the critical filling velocity increases with the increase of the mean particle diameter. It was found that the critical velocity decreases with the increase of specific energy, which is correlated with particle interlocking. It was shown that powders with high values of specific energy and high particle interlocking exhibit poorer flow properties, resulting in lower critical velocity and die filling performance. The critical velocity is also influenced by the powder permeability: lower powder permeability (higher pressure drop) results in lower critical velocity. This could be caused by fine particles: higher fines content leads to more compacted powder beds and lower air permeation. It was observed that the increase of pressure drop (estimated at $15 \mathrm{kPa}$ ) up to 2 mbar leads to a drop in critical velocity, and at higher values of pressure drop the critical velocity remains consistently low. The critical velocity increases with flow function and decreases with cohesion. There appears to be a linear relation between critical velocity and flow function, whereas in case of cohesion it seems to be a power relation. Finally, the critical velocity increases as the air sensitivity index increases (less air sensitive), indicating that powders with coarser and denser particles perform better in die filling. Overall, the critical filling velocity appears to be correlated with several powder properties and could potentially be used a single flowability descriptor, useful in predicting the powder performance in die filling. More studies are necessary to link the critical velocity to the optimal filling 
642 velocity on a rotary tablet press in the presence of suction filling, and to explore the 643 reliability of the correlations for a wider range of powders.

\section{Acknowledgements}

645 This work was partially funded by the International Fine Particle Research Institute 646 (IFPRI), and we are grateful to the constructive discussion with Michele Marigo and

647 Tim Freeman. W. Zhong would like to acknowledge the financial support of National 648 Natural Science Foundation of China (Grant No. 51605192) and Shandong Provincial 649 Natural Science Foundation (Grant No. BS2015 NJ009). H.A. Makroo wishes to thank 650 the Commonwealth Scholarship Commission in the UK for the award of a 651 Commonwealth Split Site Scholarship (Ref. No. INCN-2016-149).

657

\section{References}

${ }^{1}$ Wu, C.-Y., \& Cocks, A. C. F. (2004). Flow behaviour of powders during die filling. Powder Metallurgy, 47(2), 127-136.

2 Hjortsberg, E., \& Bergquist, B. (2002). Filling induced density variations in metal powder. Powder Metallurgy, 45(2), 146-153.

3 Schneider, Sinka, \& Cocks. (2007). Characterisation of the flow behaviour of pharmaceutical powders using a model die-shoe filling system. Powder Technology, 173(1), 59-71.

4 Jackson, S., Sinka, I. C., \& Cocks, A. C. F. (2007). The effect of suction during die fill on a rotary tablet press. European Journal of Pharmaceutics and Biopharmaceutics, 65(2), 253-256. 
${ }^{5}$ Wu, C. Y., Dihoru, L., \& Cocks, A. C. F. (2003). The flow of powder into simple and stepped dies. Powder Technology, 134(1-2), 24-39.

${ }^{6}$ Wu, C. Y., Armstrong, B., \& Vlachos, N. (2012). Characterization of powder flowability for die filling. Particulate Science and Technology, 30(4), 378-389.

${ }^{7}$ Mills, L. A., \& Sinka, I. C. (2013). Effect of particle size and density on the die fill of powders. European Journal of Pharmaceutics and Biopharmaceutics, 84(3), 642-652.

${ }^{8}$ Freeman, R., \& Fu, X. (2008). Characterisation of powder bulk, dynamic flow and shear properties in relation to die filling. Powder Metallurgy, 51(3), 196-201

${ }^{9}$ Guo, Y., Kafui, K., Wu, C., Thornton, C., \& Seville, J. (2009). A coupled DEM/CFD analysis of the effect of air on powder flow during die filling. AIChE Journal, 55(1), 4962.

${ }^{10}$ Mendez, R., Muzzio, F., \& Velazquez, C. (2010). Study of the effects of feed frames on powder blend properties during the filling of tablet press dies. Powder Technology, 200(3), 105-116.

${ }^{11}$ Faqih, A. M. N., Mehrotra, A., Hammond, S. V., \& Muzzio, F. J. (2007). Effect of moisture and magnesium stearate concentration on flow properties of cohesive granular materials. International Journal of Pharmaceutics, 336(2), 338-345.

${ }^{12}$ Peeters, E., De Beer, T., Vervaet, C., \& Remon, J. (2015). Reduction of tablet weight variability by optimizing paddle speed in the forced feeder of a high-speed rotary tablet press. Drug Development and Industrial Pharmacy, 2015, Vol.41(4), P.530539, 41(4), 530-539. 
${ }^{13}$ Van Snick, B., Grymonpré, W., Dhondt, J., Pandelaere, K., Di Pretoro, G., Remon, J. P., ... Vanhoorne, V. (2018). Impact of blend properties on die filling during tableting. International Journal of Pharmaceutics, 549(1-2), 476-488.

${ }^{14} \mathrm{Fu}$, Huck, Makein, Armstrong, Willen, \& Freeman. (2012). Effect of particle shape and size on flow properties of lactose powders. Particuology, 10(2), 203-208.

${ }^{15}$ Freeman, R. (2007). Measuring the flow properties of consolidated, conditioned and aerated powders - A comparative study using a powder rheometer and a rotational shear cell. Powder Technology, 174 (1-2), 25-33.

${ }^{16}$ Leturia, M., Benali, M., Lagarde, S., Ronga, I., \& Saleh, K. (2014). Characterization of flow properties of cohesive powders: A comparative study of traditional and new testing methods. Powder Technology, 253, 406-423.

17 Hertel, Schwarz, Kobler, Hauptstein, Steckel, \& Scherließ. (2018). Powder flow analysis: A simple method to indicate the ideal amount of lactose fines in dry powder inhaler formulations. International Journal of Pharmaceutics,535(1-2), 59-67.

${ }^{18}$ Sinka, I. C., Schneider, L. C. R., \& Cocks, A. C. F. (2004). Measurement of the flow properties of powders with special reference to die fill. International Journal of Pharmaceutics, 280(1-2), 27-38.

${ }^{19}$ Schiano, Chen, \& Wu. (2018). The effect of dry granulation on flow behaviour of pharmaceutical powders during die filling. Powder Technology, 337, 78-83.

${ }^{20}$ Leung, L. Y., Mao, C., Chen, L. P., \& Yang, C. Y. (2016). Precision of pharmaceutical powder flow measurement using ring shear tester: High variability is inherent to powders with low cohesion. Powder Technology, 301, 920-926. 
${ }^{21}$ Wang, Y., Snee, R. D., Meng, W., \& Muzzio, F. J. (2016). Predicting flow behavior of pharmaceutical blends using shear cell methodology: A quality by design approach. Powder Technology, 294, 22-29. 
Table 1. Size parameters data from QICPIC Particle Size Analyser (data expressed as mean \pm standard deviation).

\begin{tabular}{|c|c|c|c|c|c|c|c|}
\hline \multirow{2}{*}{ Name } & \multicolumn{4}{|c|}{ EQPC $(\mu \mathrm{m})$} & \multicolumn{3}{|c|}{ Sphericity } \\
\hline & $d_{10}$ & $d_{50}$ & $\mathbf{d}_{90}$ & span & $\mathbf{s}_{10}$ & $S_{50}$ & $\mathbf{S}_{90}$ \\
\hline $\begin{array}{l}\text { Lactose } \\
\text { monohydrate }\end{array}$ & $36.25 \pm 0.62$ & $84.19 \pm 2.23$ & $159.28 \pm 6.32$ & $1.46 \pm 0.44$ & $0.60 \pm 0.04$ & $0.82 \pm 0.01$ & $0.898 \pm 0.003$ \\
\hline MCC DG & $37.41 \pm 0.11$ & $80.24 \pm 0.69$ & $168.13 \pm 1.47$ & $1.63 \pm 0.47$ & $0.56 \pm 0.02$ & $0.71 \pm 0.01$ & $0.84 \pm 0.01$ \\
\hline MCC PH101 & $43.71 \pm 0.04$ & $88.08 \pm 0.03$ & $152.10 \pm 1.71$ & $1.23 \pm 0.50$ & $0.55 \pm 0.01$ & $0.65 \pm 0.01$ & $0.81 \pm 0.01$ \\
\hline Mannitol & $85.73 \pm 0.89$ & $125.38 \pm 0.69$ & $184.82 \pm 6.42$ & $0.79 \pm 0.69$ & $0.67 \pm 0.01$ & $0.81 \pm 0.001$ & $0.90 \pm 0.01$ \\
\hline DCPA & $95.85 \pm 4.77$ & $202.22 \pm 3.71$ & $279.60 \pm 1.98$ & $0.91 \pm 0.47$ & $0.70 \pm 0.01$ & $0.78 \pm 0.01$ & $0.84 \pm 0.01$ \\
\hline MCC CP102 & $143.84 \pm 0.59$ & $186.60 \pm 0.9$ & $221.40 \pm 2.14$ & $0.42 \pm 0.77$ & $0.85 \pm 0.01$ & $0.91 \pm 0.01$ & $0.94 \pm 0.01$ \\
\hline MCC PH102 & $48.64 \pm 1.89$ & $108.62 \pm 3.69$ & $222.33 \pm 3.14$ & $1.60 \pm 0.45$ & $0.58 \pm 0.01$ & $0.71 \pm 0.01$ & $0.82 \pm 0.01$ \\
\hline
\end{tabular}


Table 2. Mass flow rate and flow indices evaluated with Flodex (data expressed as mean \pm standard deviation).

\begin{tabular}{|l|c|c|}
\hline \multirow{2}{*}{ Material } & Mass flow rate through an orifice & Flow index \\
& diameter of $\mathbf{2 4} \mathbf{~ m m}$ & (Orifice diameter, $\mathbf{m m})$ \\
\cline { 2 - 3 } & $(\mathbf{g} / \mathbf{s})$ & 24 \\
\hline Lactose monohydrate & $55.3 \pm 5.7$ & 22 \\
\hline MCC DG & $60.0 \pm 5.0$ & 20 \\
\hline MCC PH101 & $47.5 \pm 4.4$ & 4 \\
\hline Mannitol & $130.7 \pm 2.4$ & \\
\hline DCPA & $140.7 \pm 2.0$ & 4 \\
\hline MCC CP102 & $64.9 \pm 1.0$ & \\
\hline MCC PH102 & $45.5 \pm 0.8$ & 5 \\
\hline
\end{tabular}


Table 3. Specific energy and pressure drop for the examined powders (data expressed as mean \pm standard deviation).

\begin{tabular}{|l|c|c|}
\hline \multirow{2}{*}{ Name } & Specific Energy & $\begin{array}{c}\text { Pressure drop at 15 kPa normal } \\
\text { stress }\end{array}$ \\
\cline { 2 - 3 } & $(\mathbf{m J} / \mathbf{g})$ & (mbar) \\
\hline Lactose monohydrate & $9.33 \pm 0.58$ & $9.30 \pm 0.02$ \\
\hline MCC DG & $8.05 \pm 1.42$ & $3.01 \pm 0.03$ \\
\hline MCC PH101 & $10.06 \pm 0.06$ & $1.41 \pm 0.04$ \\
\hline Mannitol & $5.76 \pm 0.07$ & $0.91 \pm 0.02$ \\
\hline DCPA & $6.28 \pm 0.02$ & $0.86 \pm 0.01$ \\
\hline MCC CP102 & $4.52 \pm 0.03$ & $0.66 \pm 0.02$ \\
\hline MCC PH102 & $7.86 \pm 0.09$ & $1.41 \pm 0.04$ \\
\hline
\end{tabular}


Table 4. Flow function and cohesion determined in the shear cell (data expressed as mean \pm standard deviation).

\begin{tabular}{|l|c|c|}
\hline \multirow{2}{*}{ Material } & Flow Function (ffc) & Cohesion \\
\cline { 2 - 3 } & $(-)$ & $\mathbf{( k P a )}$ \\
\hline Lactose monohydrate & $3.38 \pm 0.22$ & $0.44 \pm 0.03$ \\
\hline MCC DG & $10.04 \pm 3.56$ & $0.17 \pm 0.06$ \\
\hline MCC PH101 & $7.24 \pm 1.96$ & $0.23 \pm 0.06$ \\
\hline Mannitol & $20.92 \pm 2.08$ & $0.06 \pm 0.01$ \\
\hline DCPA & $37.76 \pm 9.21$ & $0.03 \pm 0.01$ \\
\hline MCC CP102 & $14.44 \pm 1.50$ & $0.09 \pm 0.01$ \\
\hline MCC PH102 & $17.96 \pm 4.36$ & $0.08 \pm 0.02$ \\
\hline
\end{tabular}


Table 5. True density, d50 and air sensitivity index $\xi$ for all powders considered (data expressed as mean \pm standard deviation).

\begin{tabular}{|l|c|c|c|}
\hline \multirow{2}{*}{ Name } & $\boldsymbol{\rho}_{\mathbf{s}}$ & $\mathbf{d}_{50}$ & $\xi$ \\
\cline { 2 - 4 } & $\mathbf{( k g / \mathbf { m } ^ { 3 } )}$ & $(\boldsymbol{\mu m})$ & $(-)$ \\
\hline Lactose monohydrate & 1540 & $84.19 \pm 2.23$ & $4.33 \times 10^{4}$ \\
\hline MCC DG & 1786 & $88.08 \pm 0.03$ & $5.08 \times 10^{4}$ \\
\hline MCC PH101 & 1581 & $80.24 \pm 0.69$ & $5.27 \times 10^{4}$ \\
\hline Mannitol & 1469 & $125.38 \pm 0.69$ & $1.31 \times 10^{5}$ \\
\hline DCPA & 2300 & $202.22 \pm 3.71$ & $1.35 \times 10^{6}$ \\
\hline MCC CP102 & 1600 & $186.60 \pm 0.9$ & $5.13 \times 10^{5}$ \\
\hline MCC PH102 & 1570 & $108.62 \pm 3.69$ & $9.74 \times 10^{4}$ \\
\hline
\end{tabular}


Table 6. Critical filling velocity and exponent $\mathrm{n}$ for all powders considered.

\begin{tabular}{|l|c|c|}
\hline \multirow{2}{*}{ Name } & Critical filling velocity & $\mathbf{n}$ (exponent) \\
\cline { 2 - 3 } & $(\mathbf{m m} / \mathbf{s})$ & 1.2 \\
\hline Lactose monohydrate & 18 & 1.2 \\
\hline MCC DG & 25 & 0.97 \\
\hline MCC PH101 & 28 & 0.92 \\
\hline Mannitol & 77.2 & 1.25 \\
\hline DCPA & 128.5 & 1.05 \\
\hline MCC CP102 & 148.2 & 0.86 \\
\hline MCC PH102 & 50 & $(-)$ \\
\hline
\end{tabular}



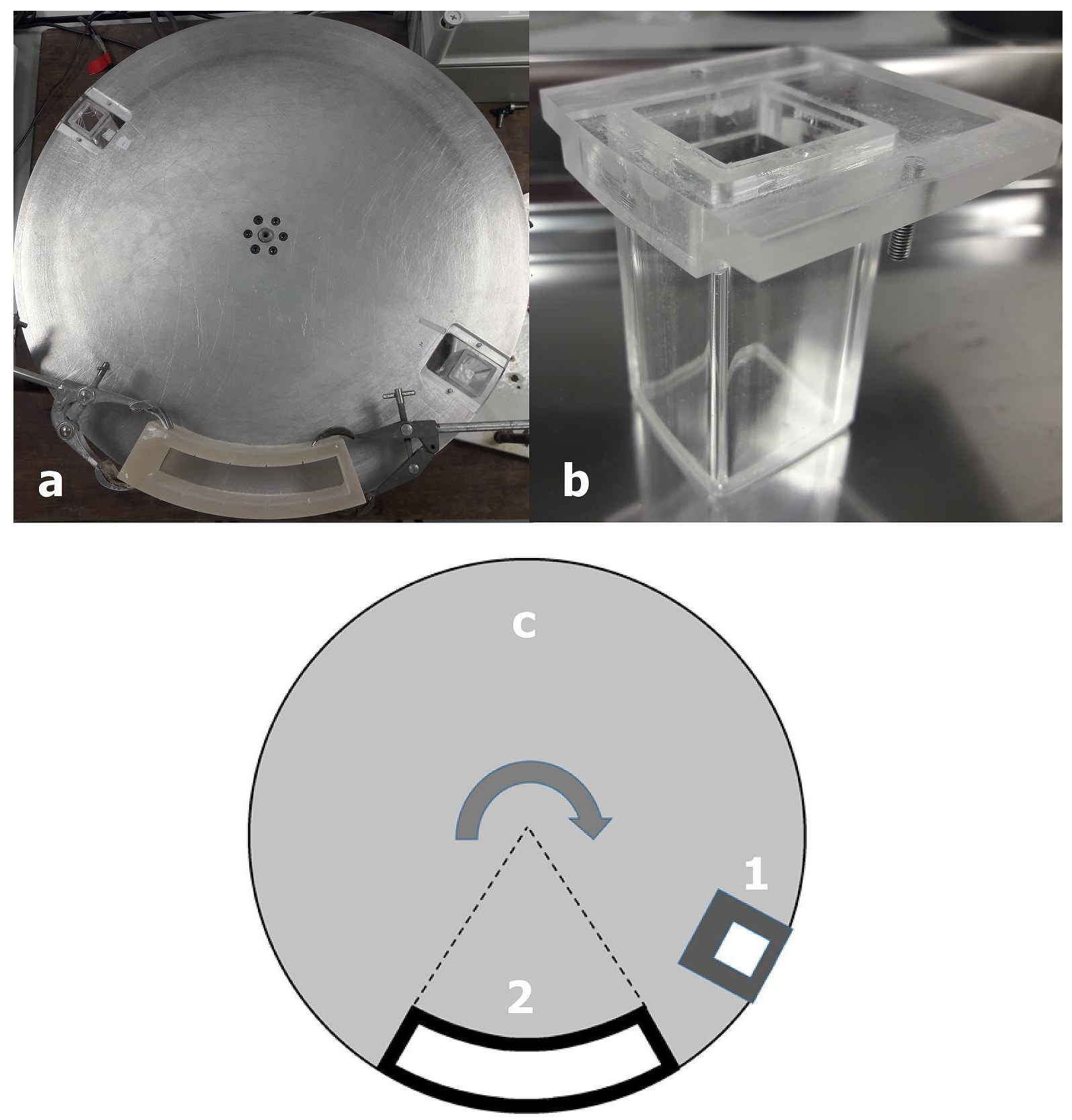

Fig. 1 The rotary die filling device used in this study: (a) top view, (b) the die and (c) the schematic diagram of the device, where 1 - the die, 2 - the feeder shoe). 


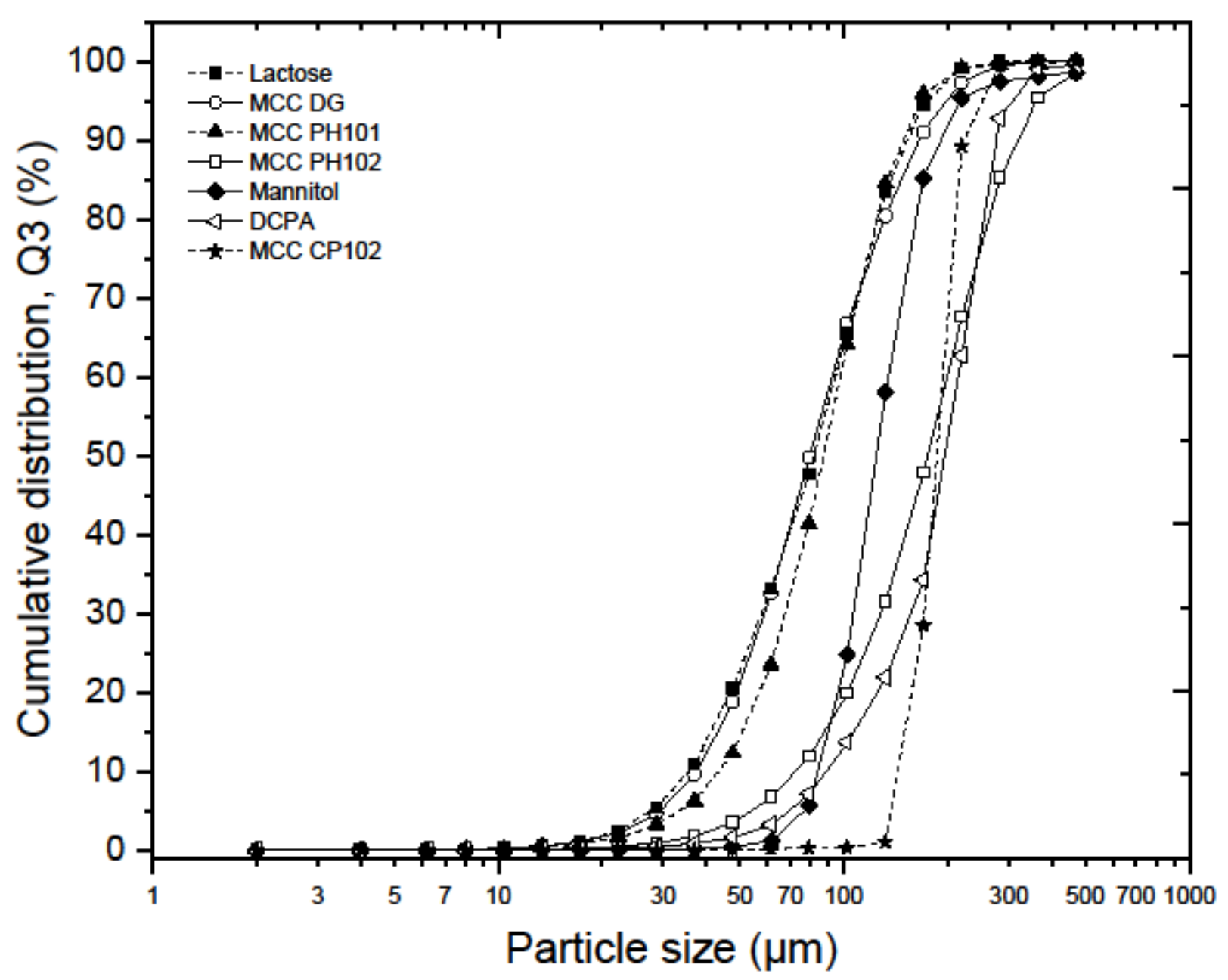

Fig. 2 Cumulative particle size distributions for all powders considered. 


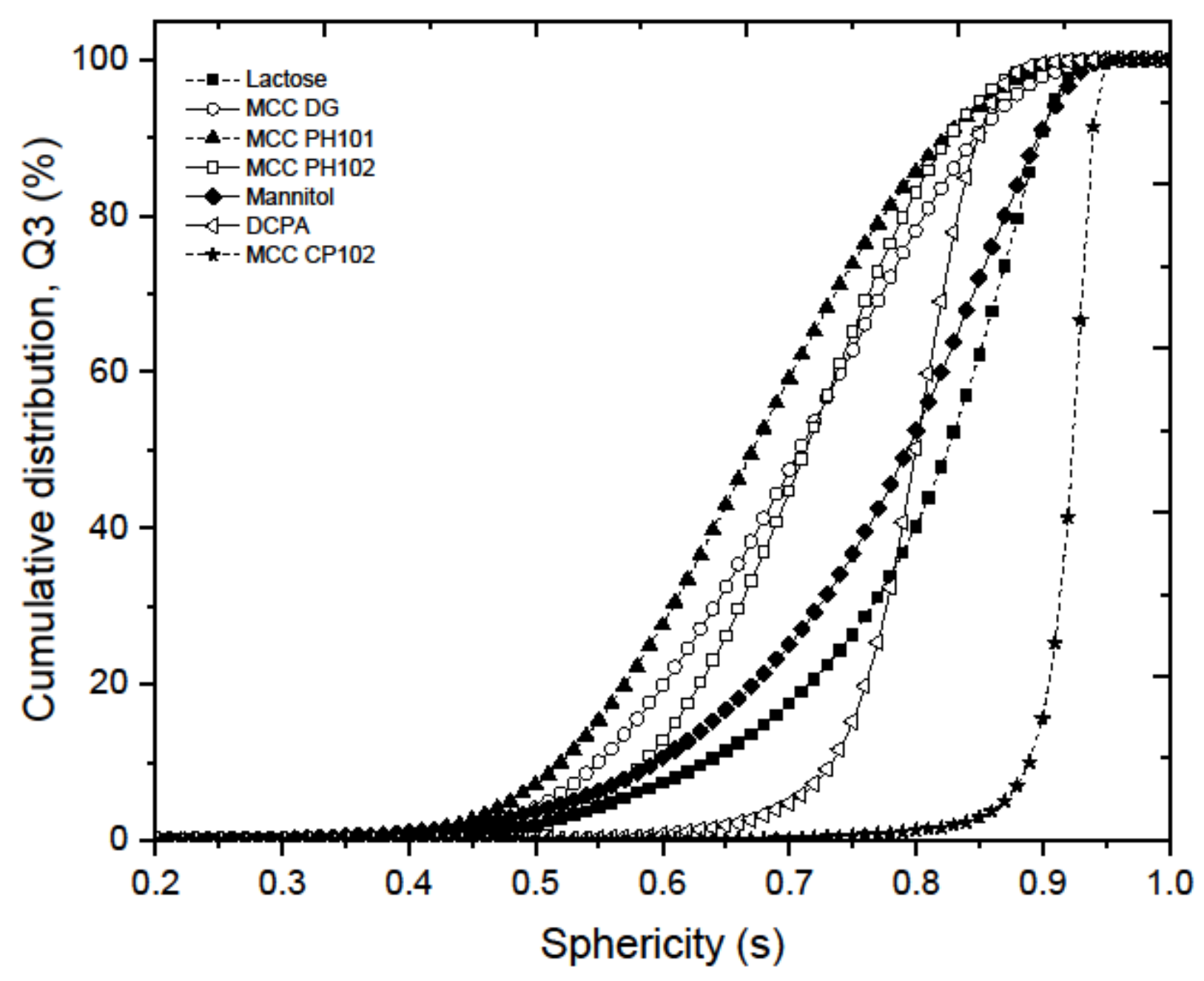

Fig. 3 Cumulative sphericity distributions for all powders considered. 


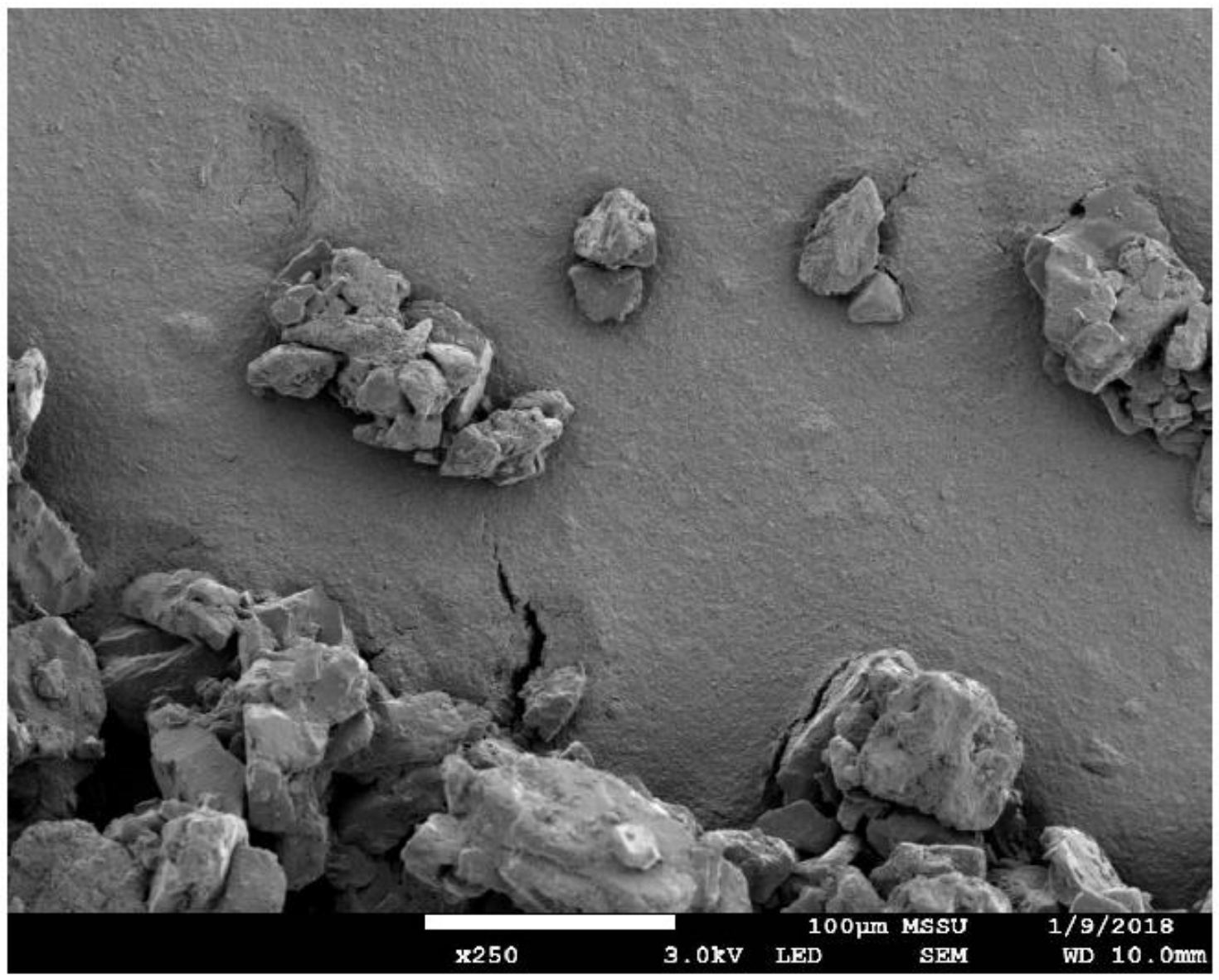

Fig. 4A Scanning electron microscope image of lactose monohydrate. 


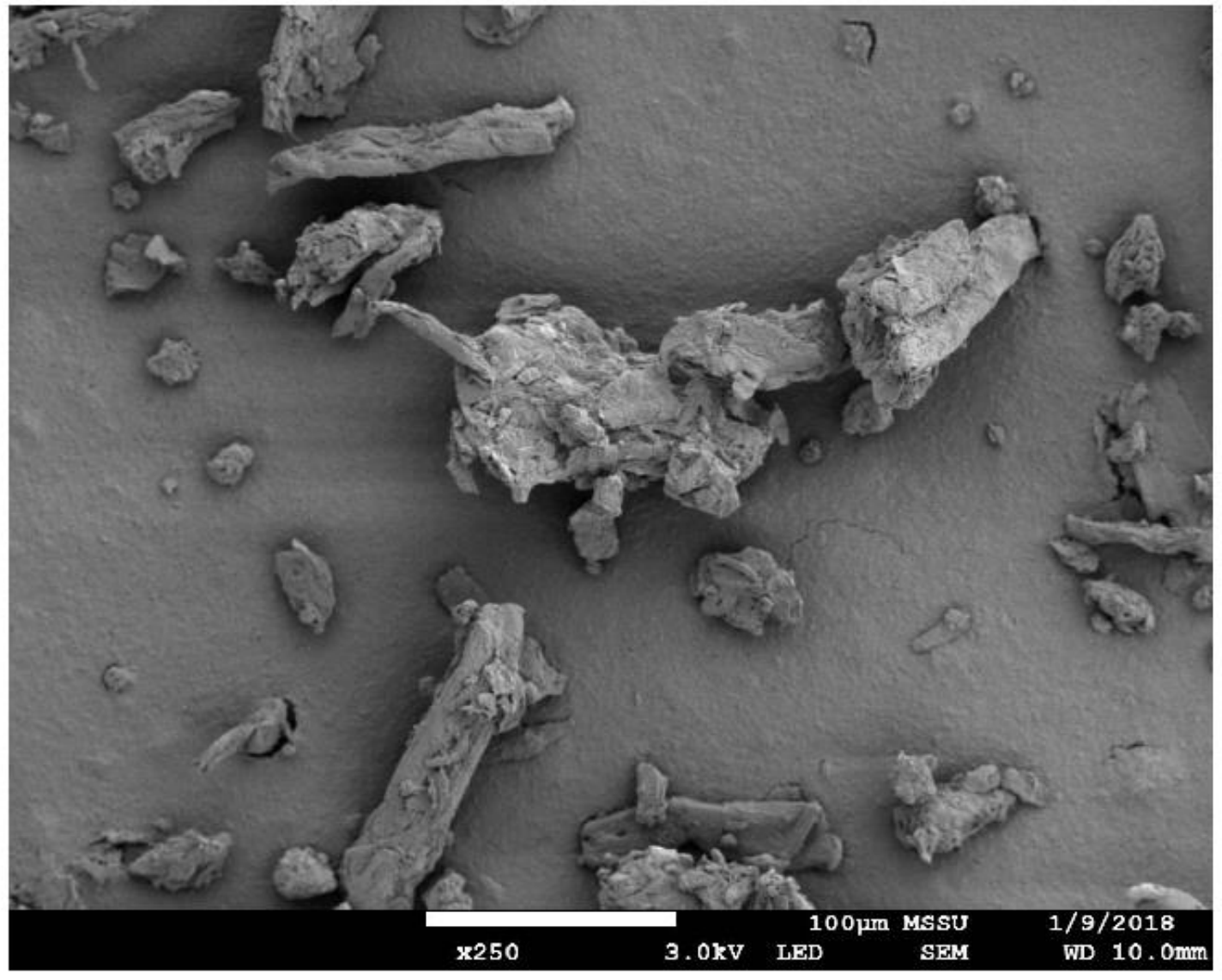

Fig. 4B Scanning electron microscope image of MCC DG. 


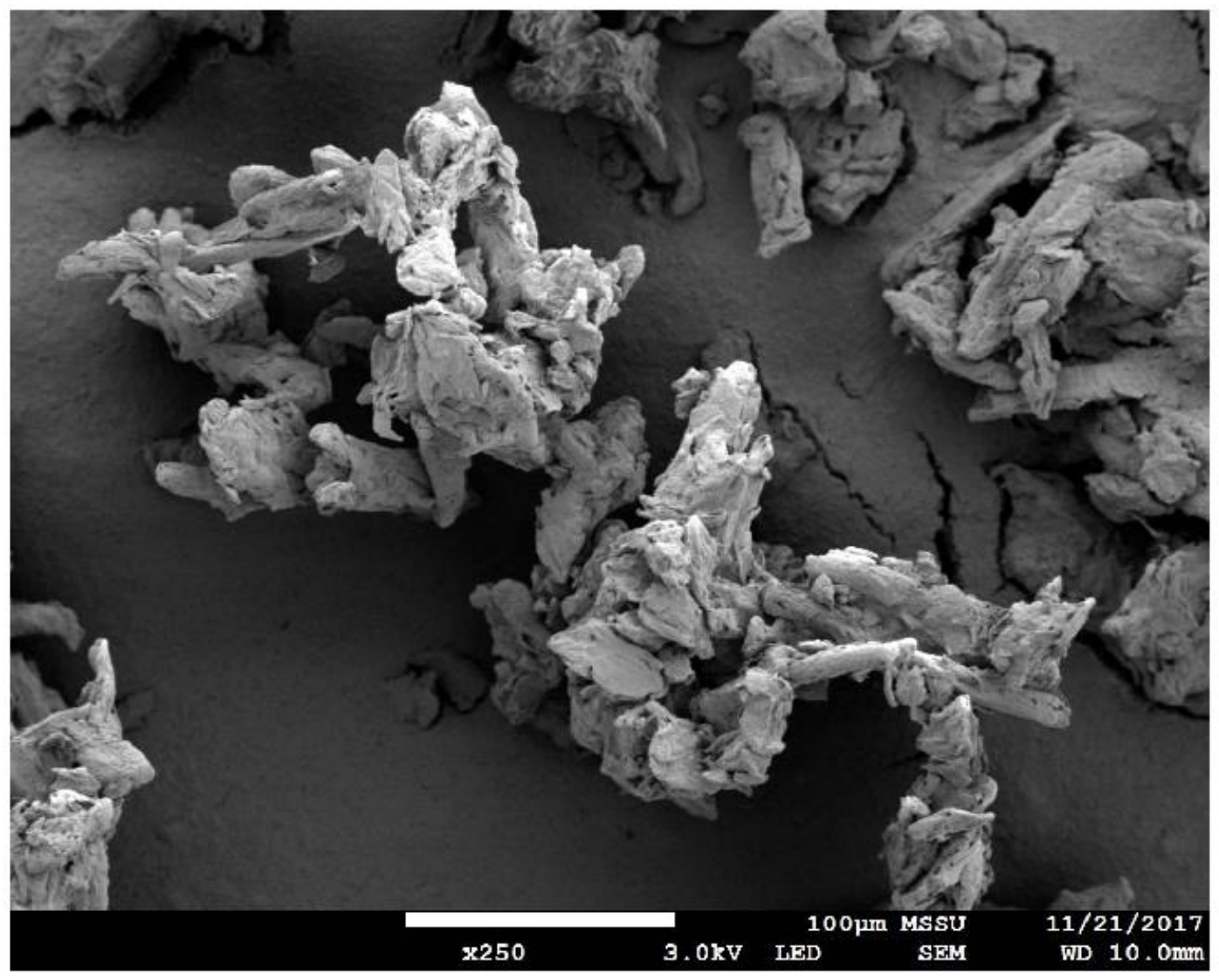

Fig. 4C Scanning electron microscope image of MCC PH101 


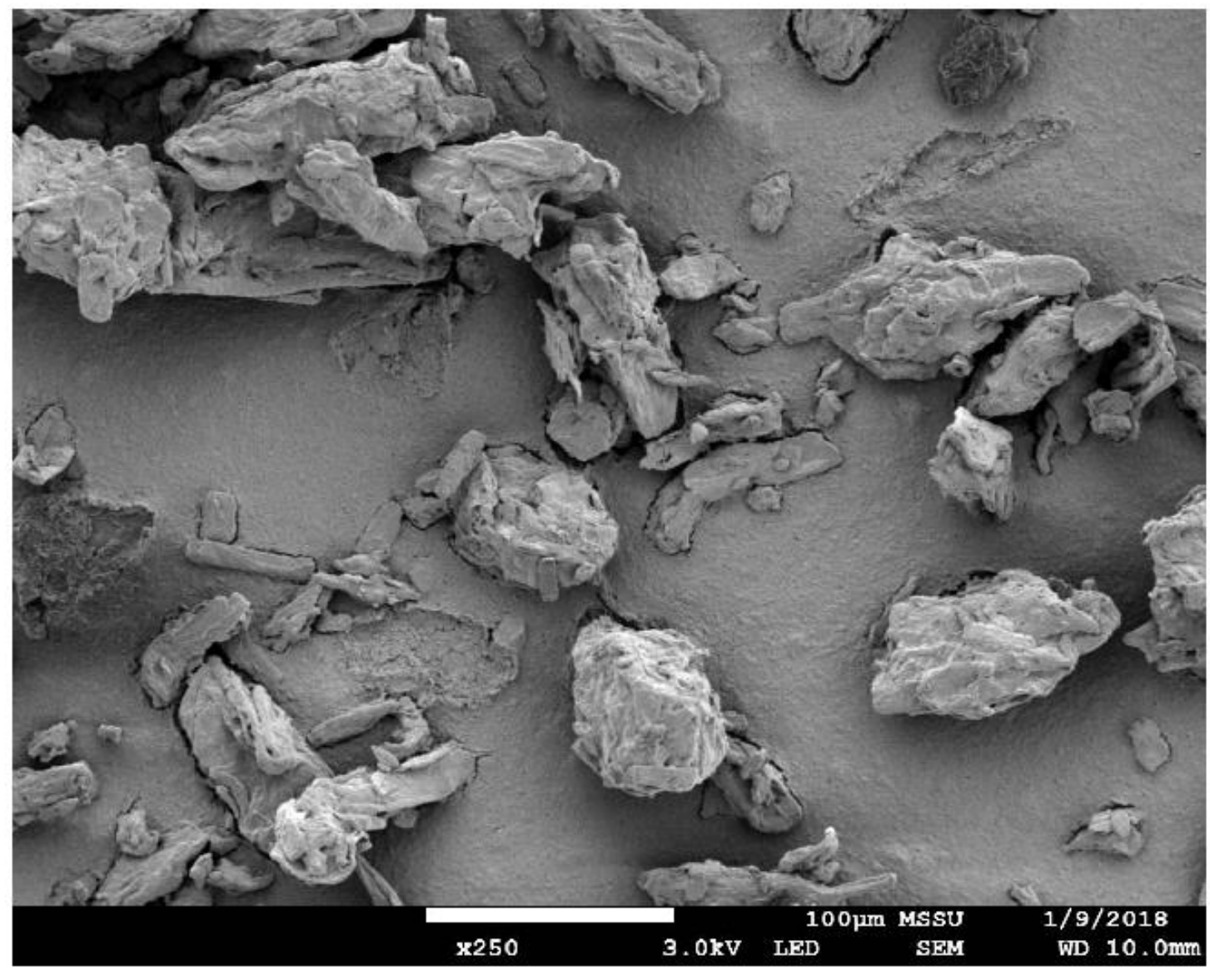

Fig. 4D Scanning electron microscope image of MCC PH102. 


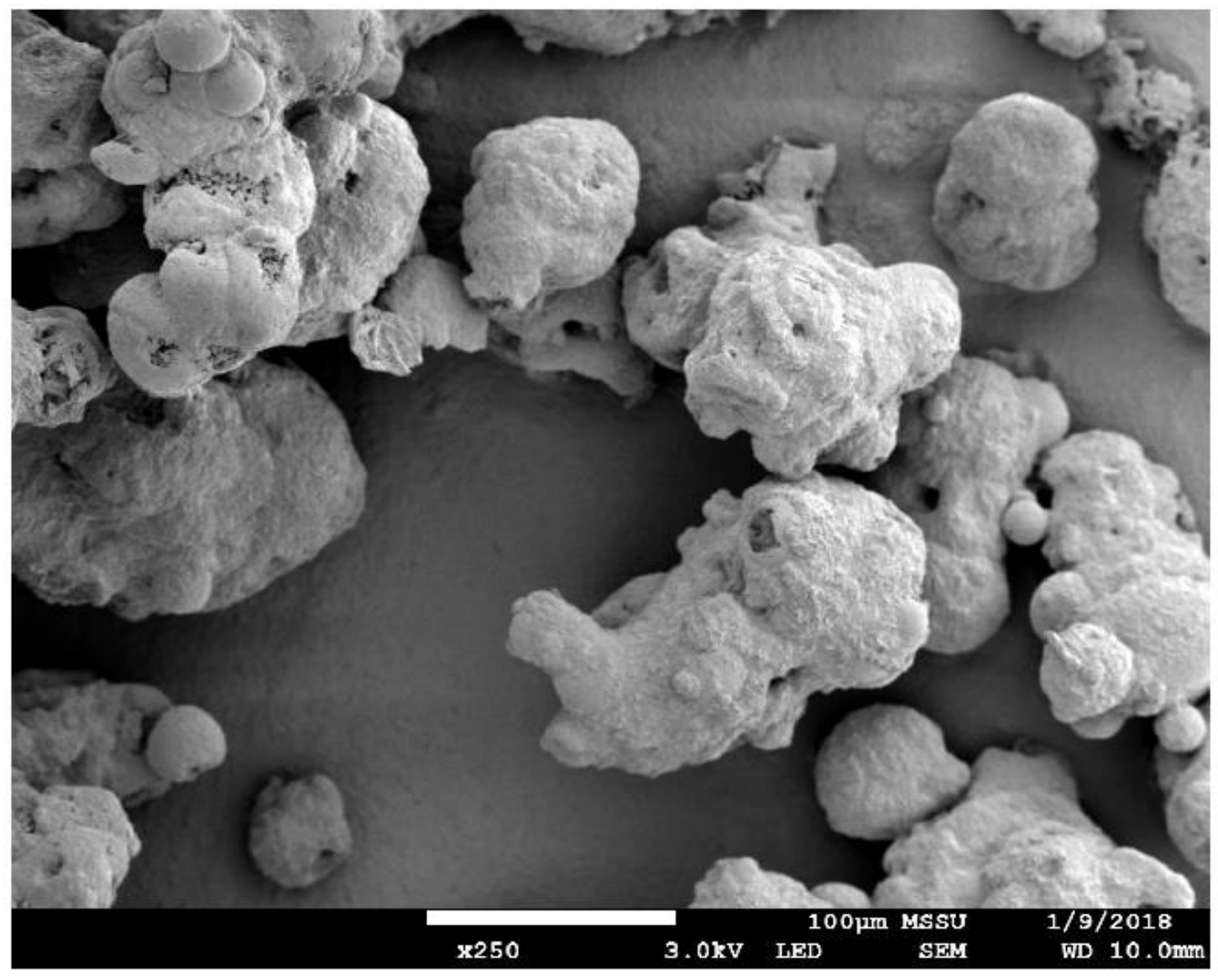

Fig. 4E Scanning electron microscope image of mannitol. 


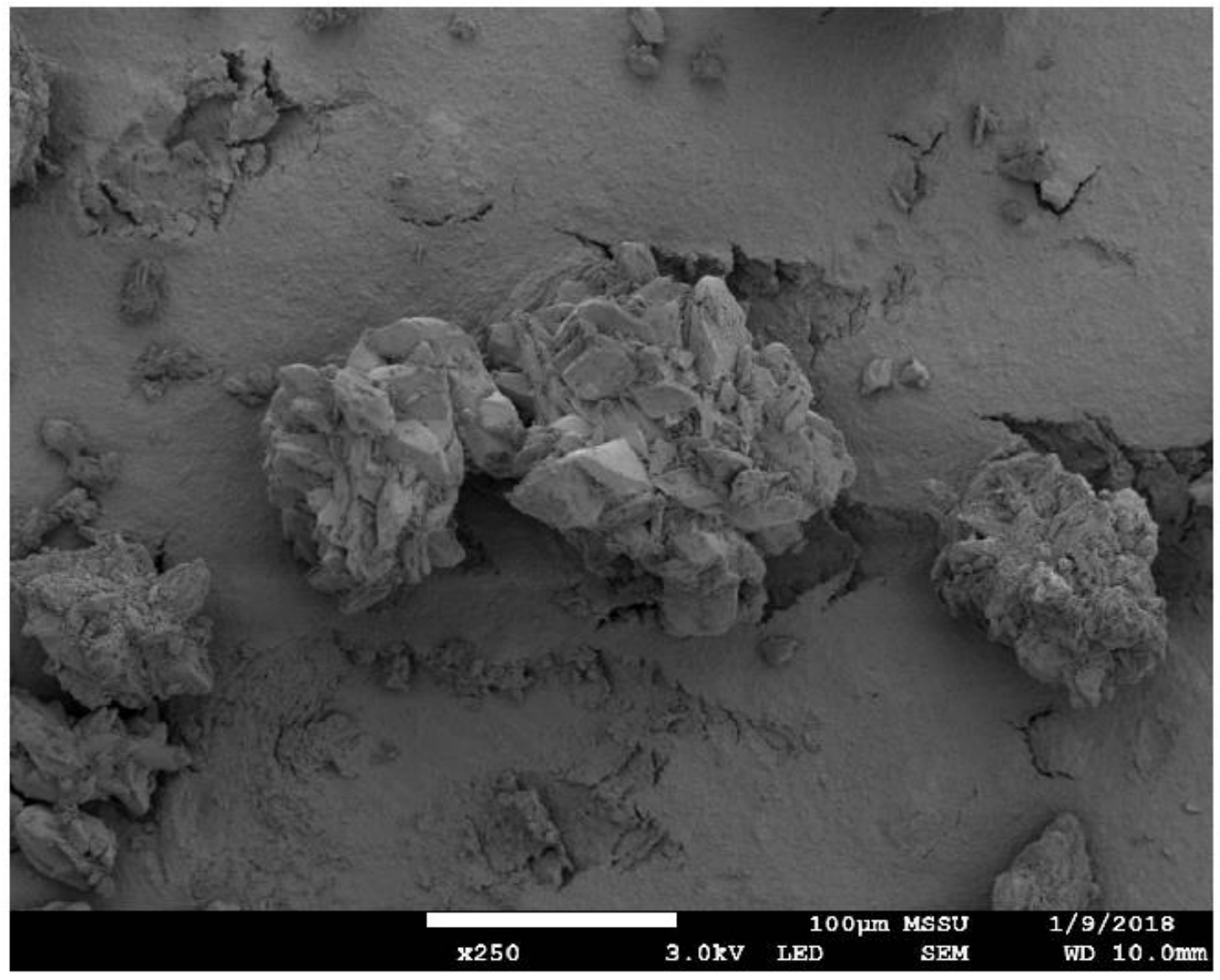

Fig. 4F Scanning electron microscope image of DCPA. 


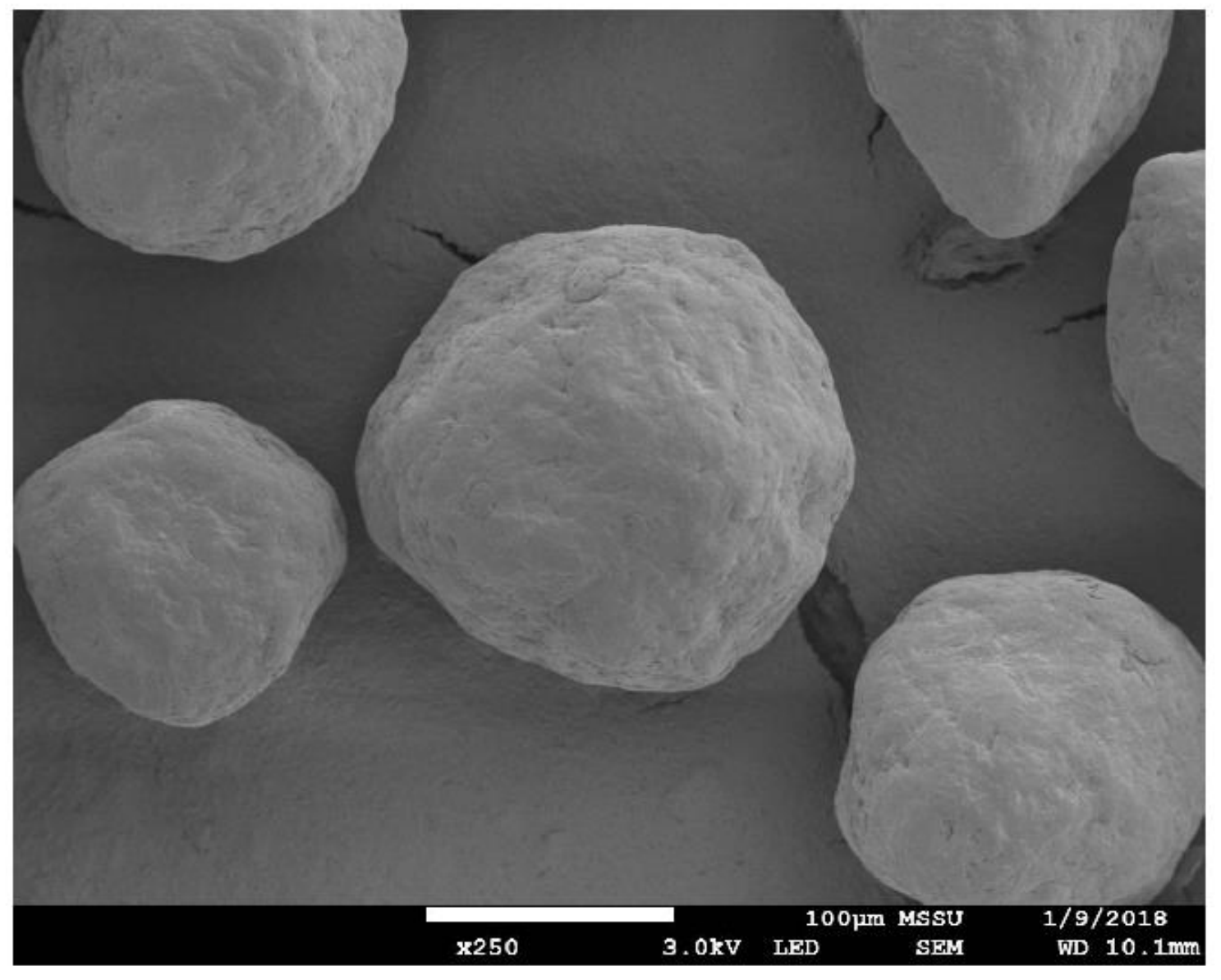

Fig. 4G Scanning electron microscope image of MCC CP102. 


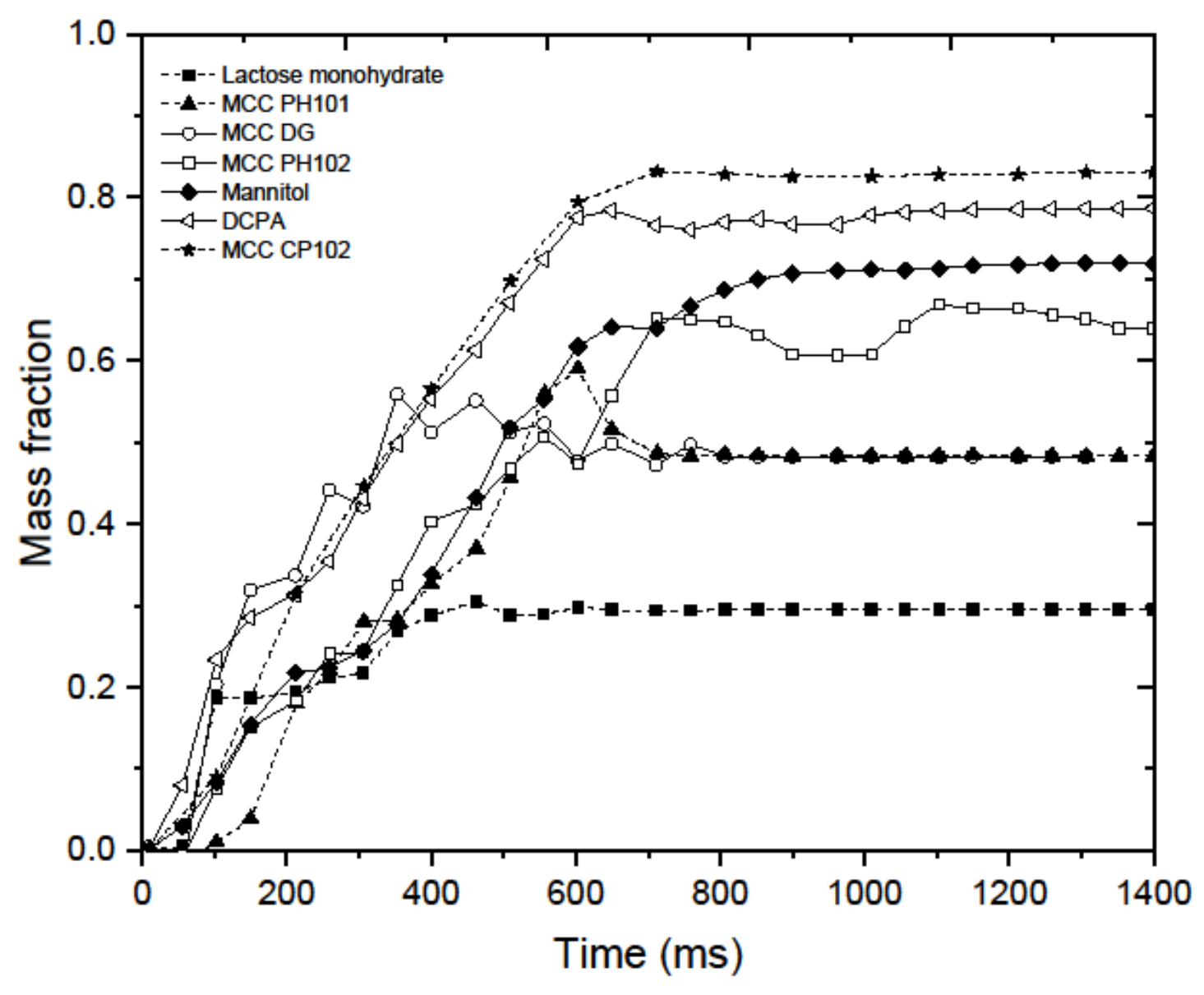

Fig. 5 Variation of mass fraction of discharged powders with time. 


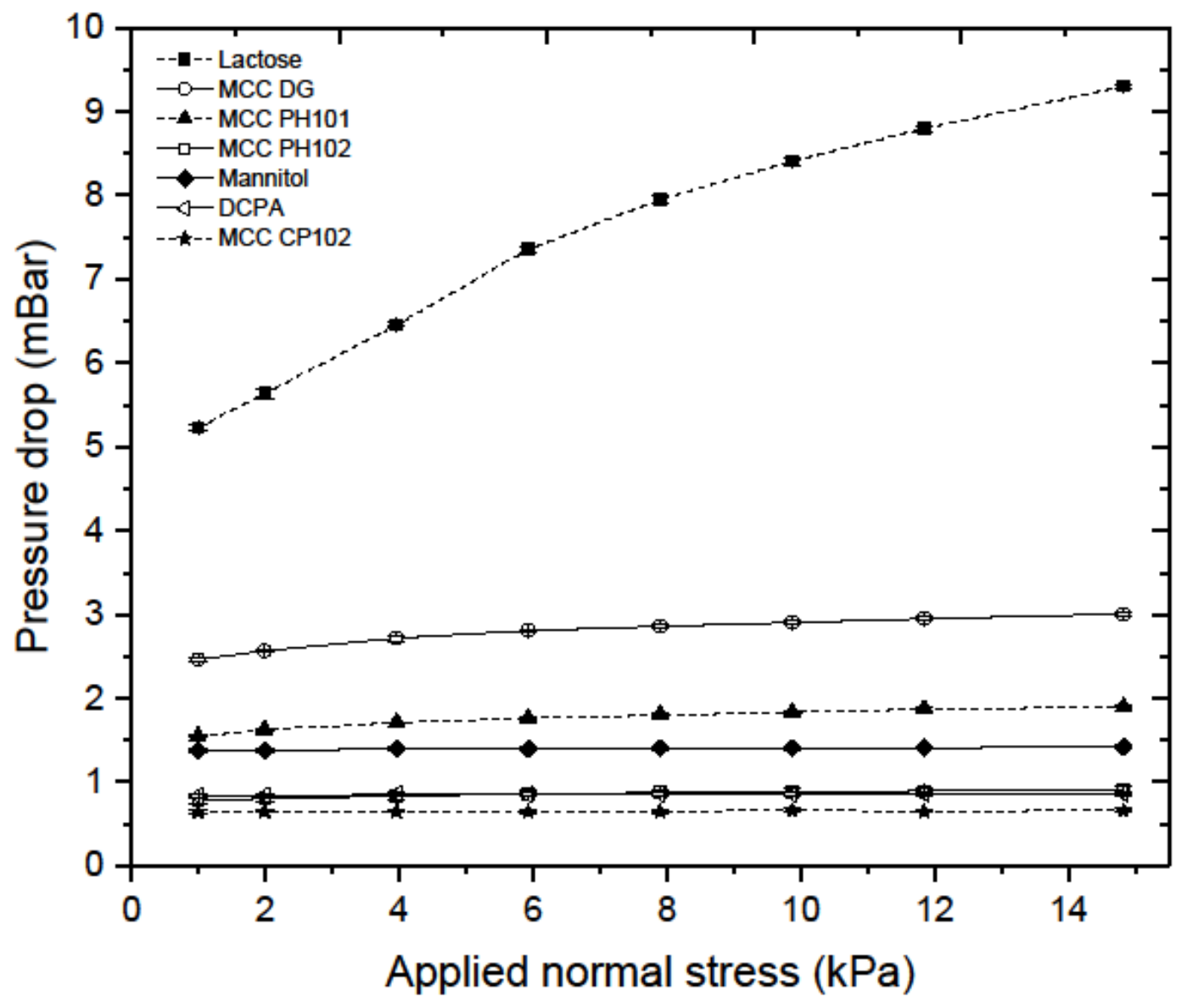

Fig. 6 Pressure drop across the powder bed as a function of applied normal stress. 


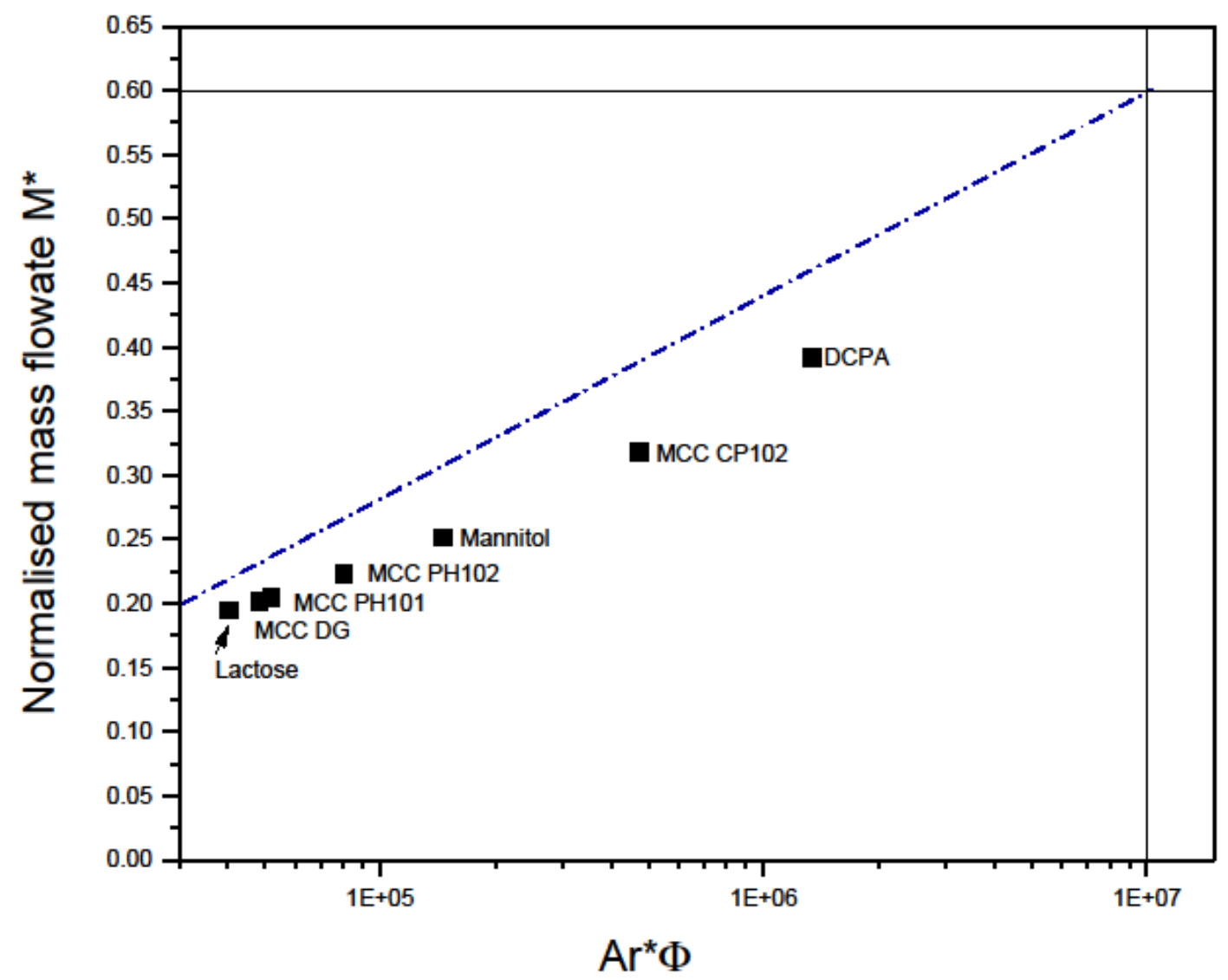

Fig. 7 Normalised mass flow rate as a function of Air sensitivity index $\left(\xi=\operatorname{Ar}{ }^{\star} \Phi\right)$ of the characterized powders. 

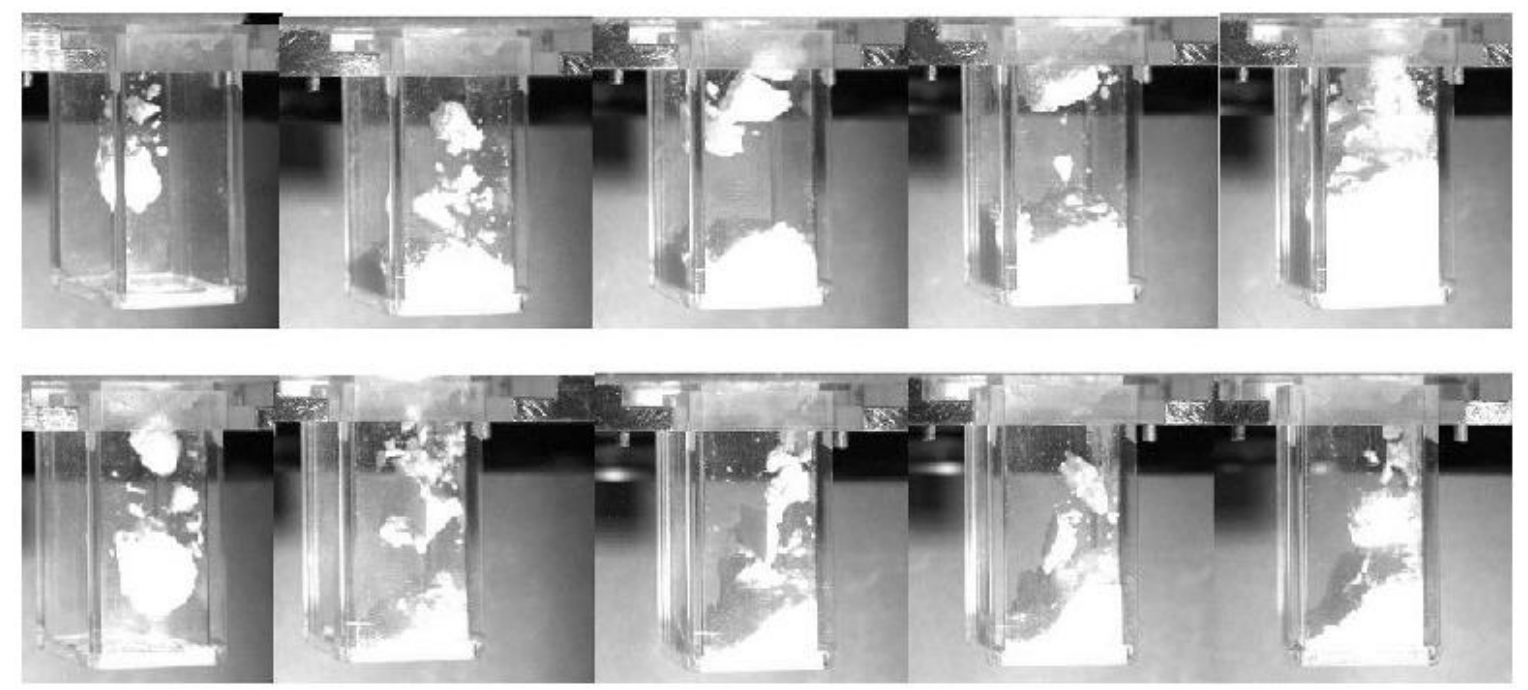

Fig. 8A Die filling with Lactose monohydrate at different velocities: (top row) -10 $\mathrm{mm} / \mathrm{s}$; (bottom row) $-70 \mathrm{~mm} / \mathrm{s}$. 

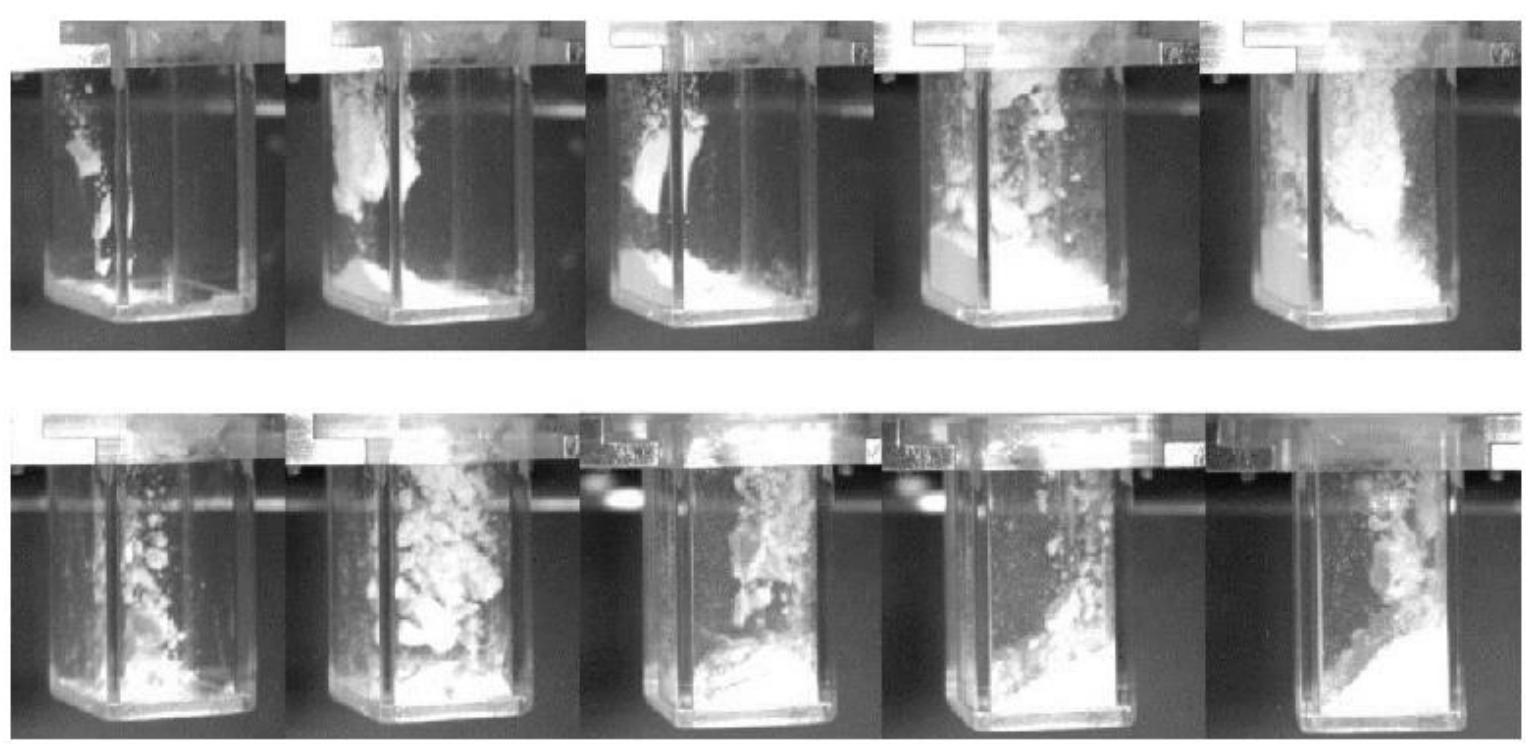

Fig. 8B Die filling with MCC DG at different velocities: (top row) $-6 \mathrm{~mm} / \mathrm{s}$; (bottom row) $-60 \mathrm{~mm} / \mathrm{s}$. 

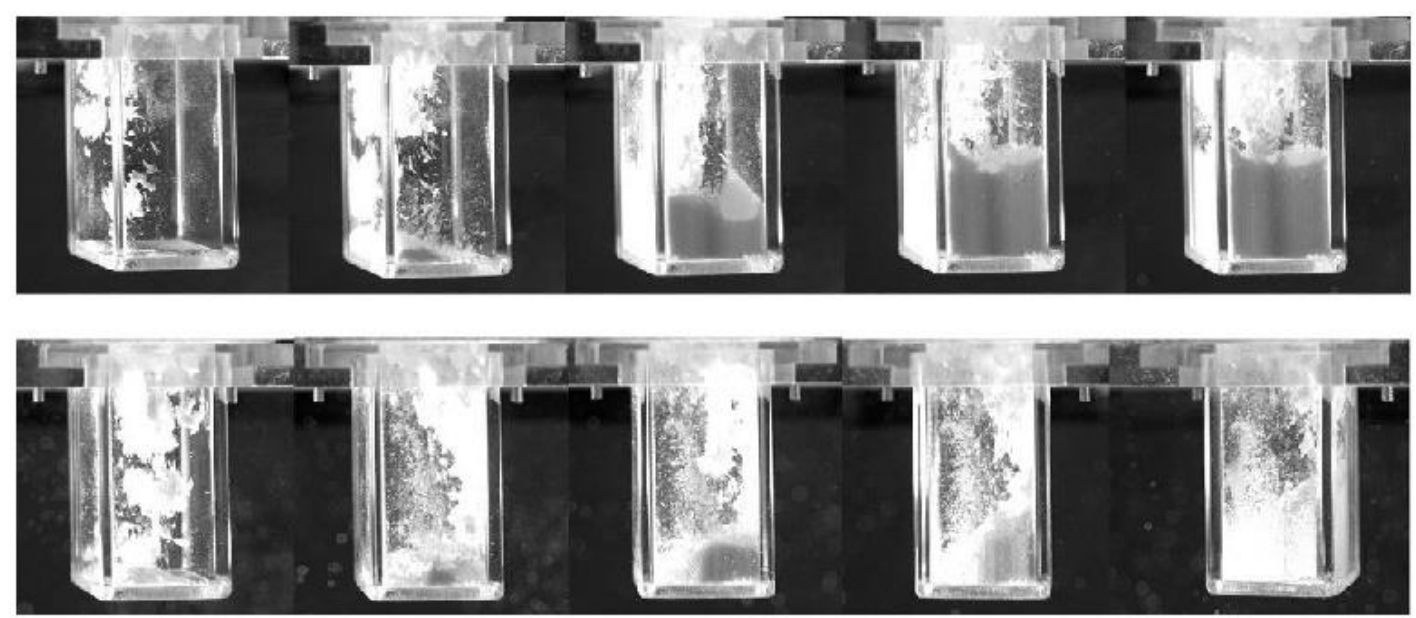

Fig. 8C Die filling with MCC PH101 at different velocities: (top row) $-20 \mathrm{~mm} / \mathrm{s}$; (bottom row) $-100 \mathrm{~mm} / \mathrm{s}$. 

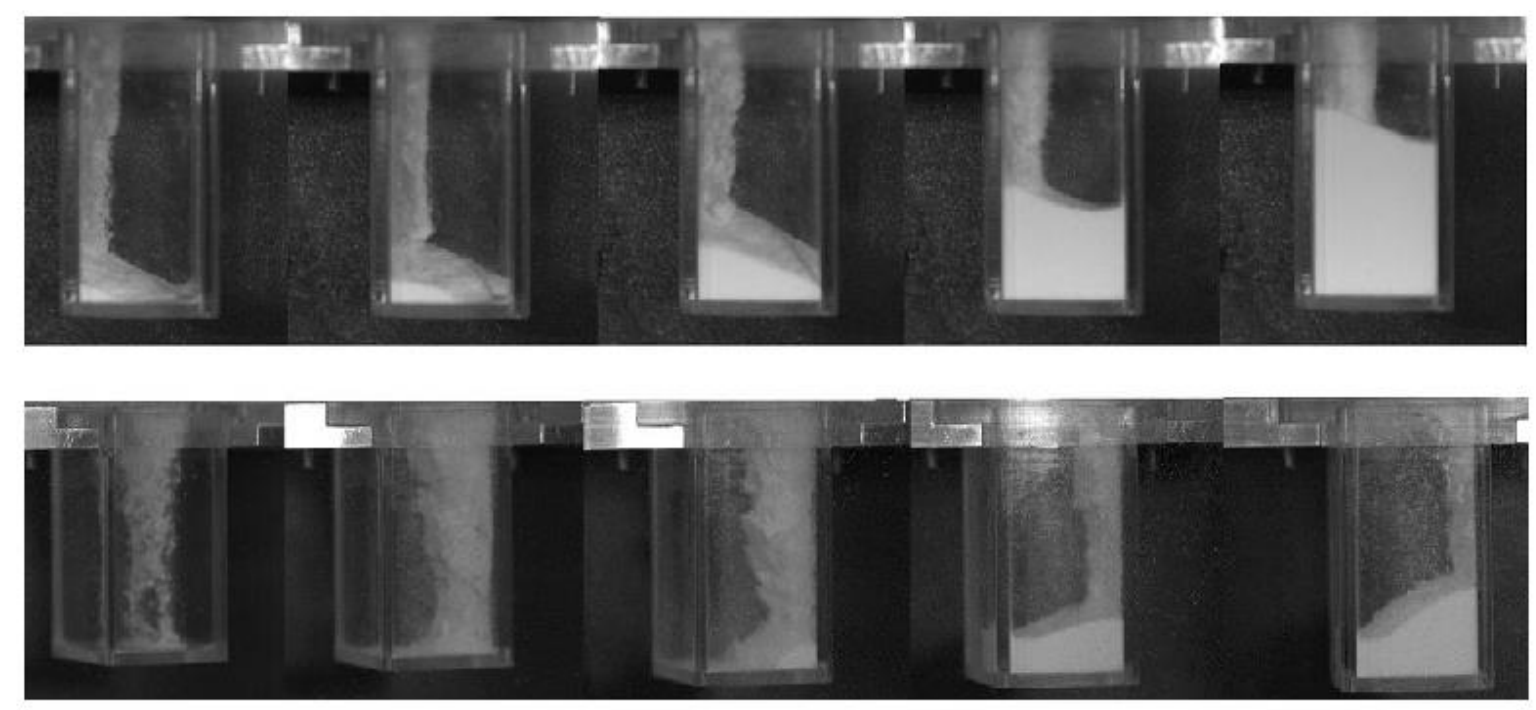

Fig. 8D Die filling with MCC PH102 at different velocities: (top row) $-50 \mathrm{~mm} / \mathrm{s}$; (bottom row) $-180 \mathrm{~mm} / \mathrm{s}$. 

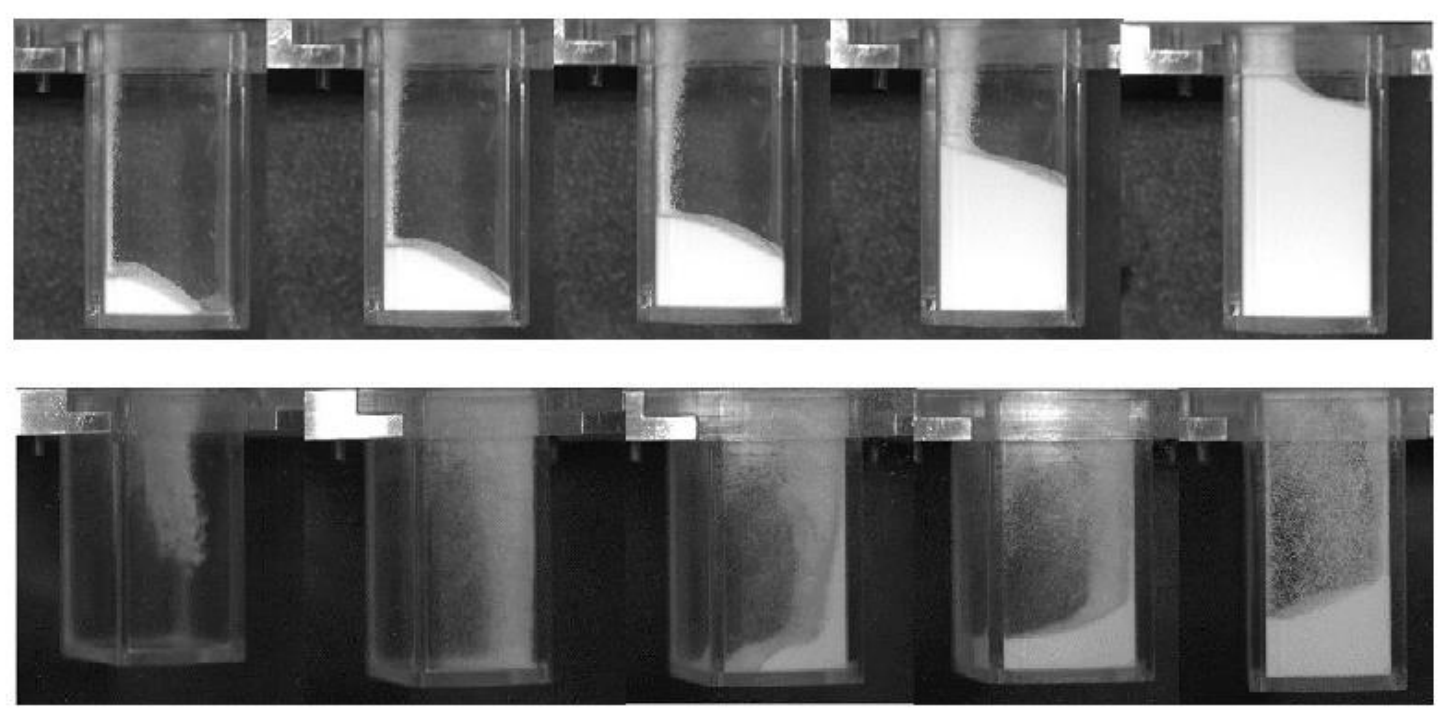

Fig. 8E Die filling with mannitol at different velocities: (top row) $-90 \mathrm{~mm} / \mathrm{s}$; (bottom row) $-250 \mathrm{~mm} / \mathrm{s}$. 

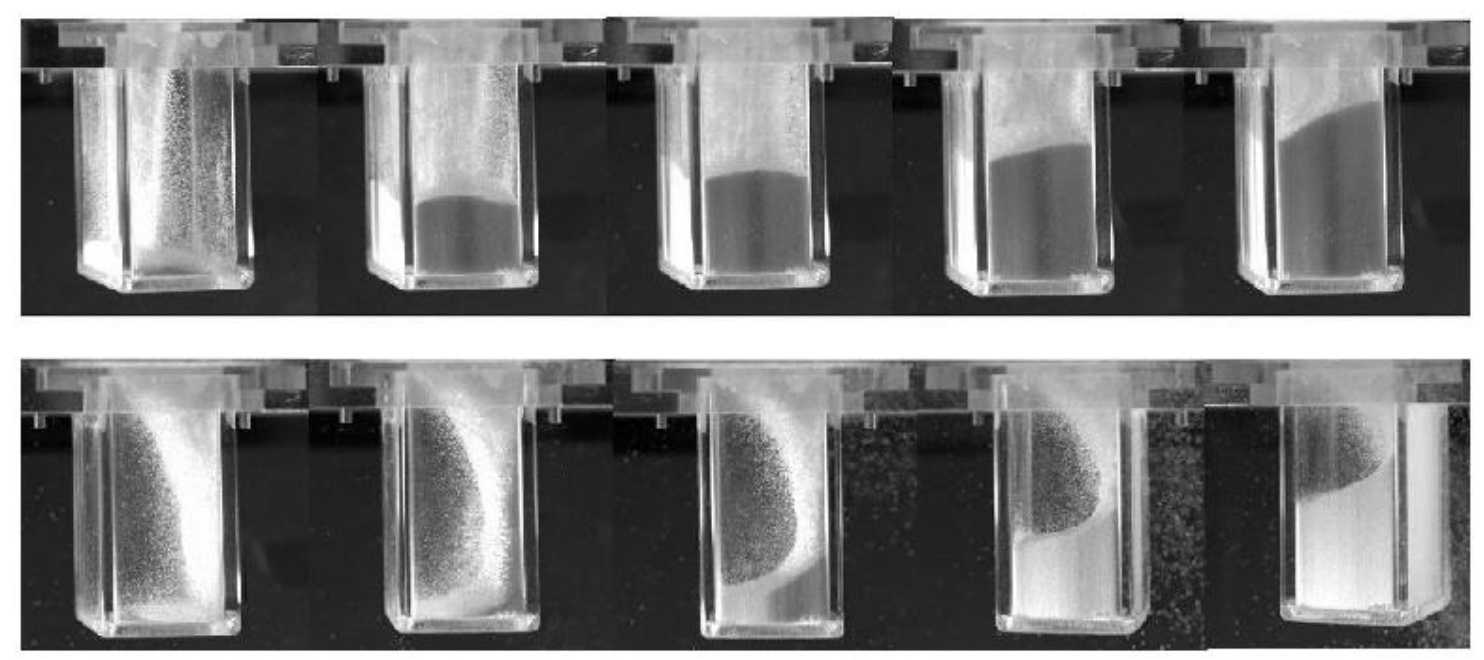

Fig. 8F Die filling with DCPA at different velocities: (top row) $-60 \mathrm{~mm} / \mathrm{s}$; (bottom row) $-240 \mathrm{~mm} / \mathrm{s}$. 

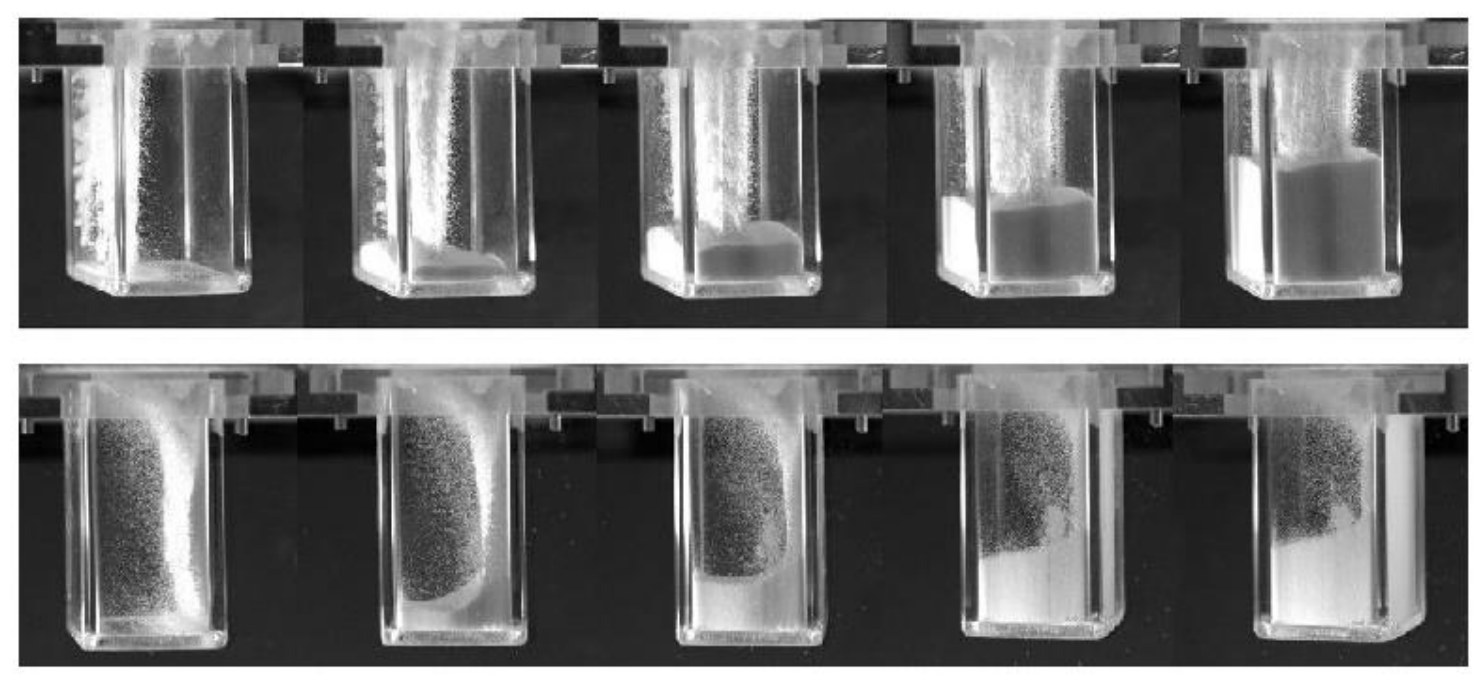

Fig. 8G Die filling with MCC CP102 at different velocities: (top row) $-60 \mathrm{~mm} / \mathrm{s}$; (bottom row) $-240 \mathrm{~mm} / \mathrm{s}$. 


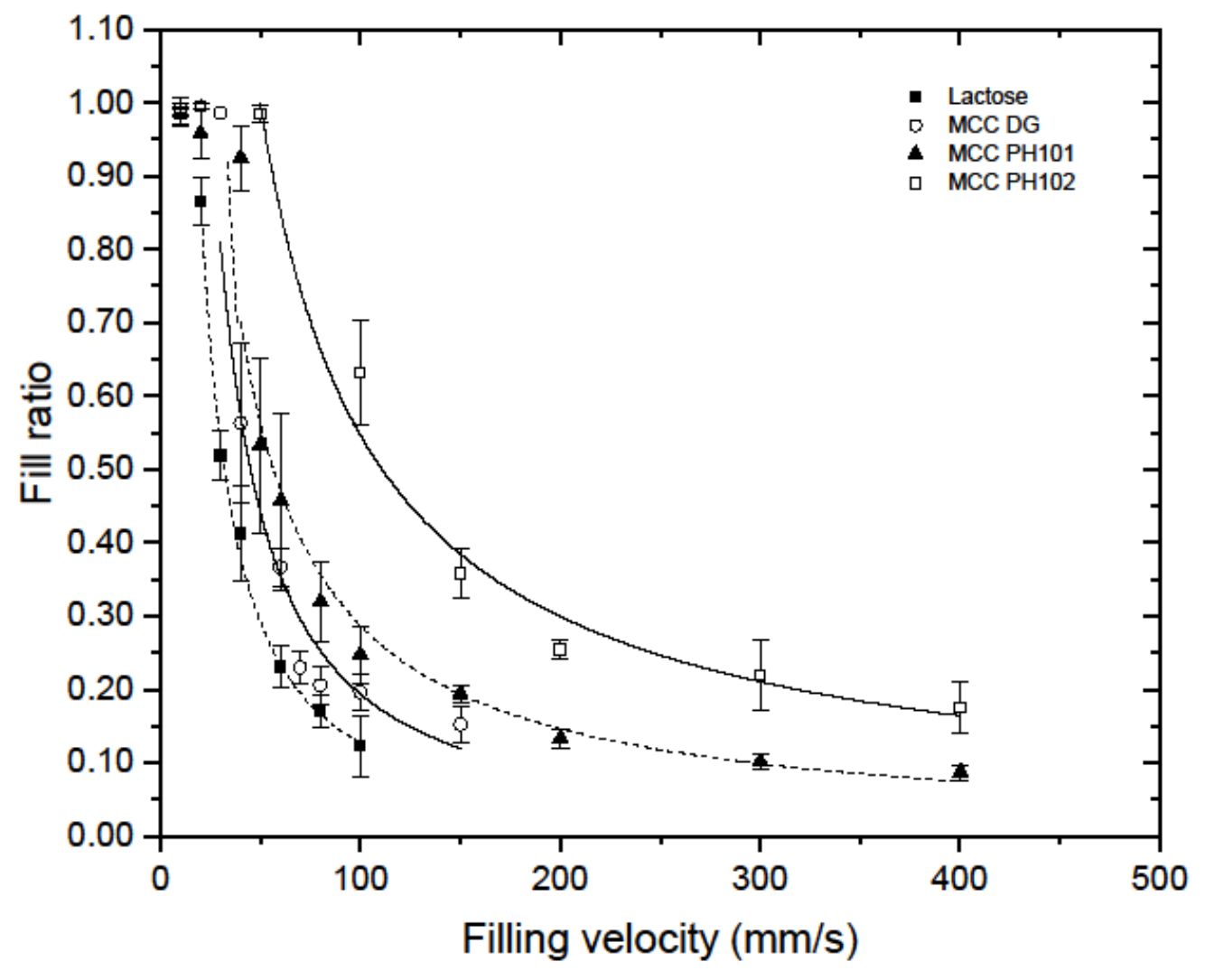

Fig. 9A Fill ratio as a function of filling velocity for cohesive powders: lactose, MCC DG, MCC PH101 (group 1) and MCC PH102. 


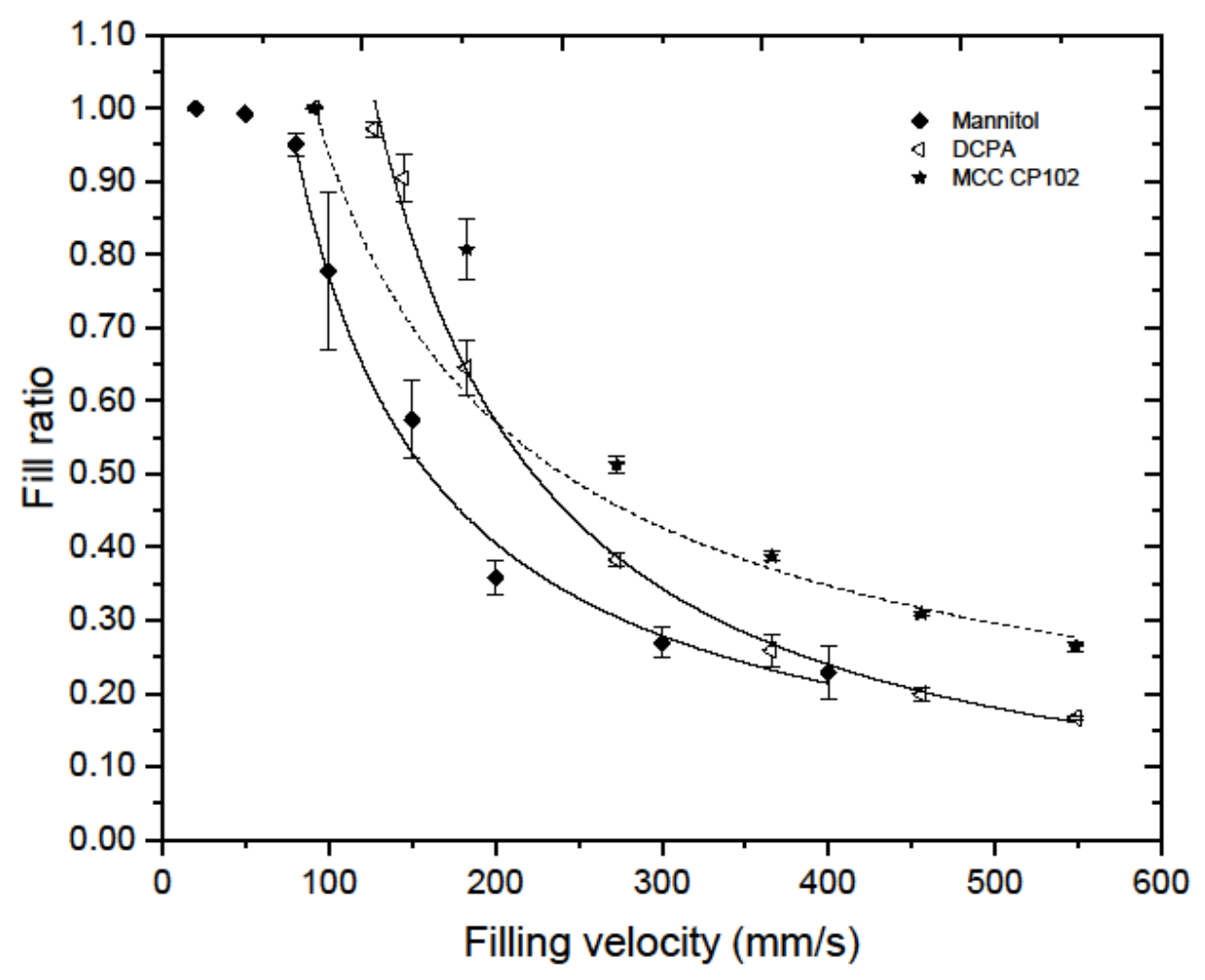

Fig. 9B Fill ratio as a function of filling velocity for freely flowing powders (group 2): mannitol, DCPA and MCC CP102. 


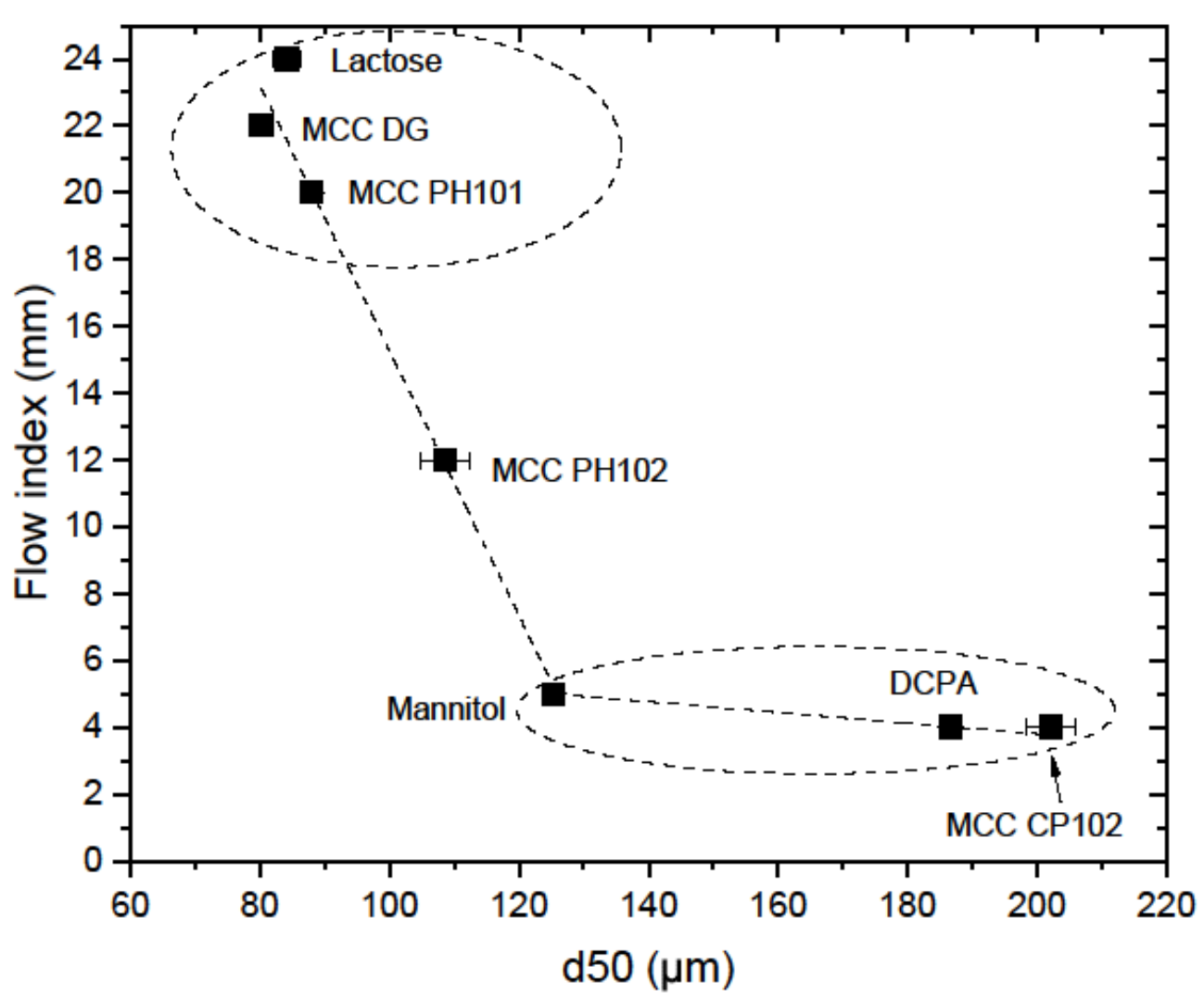

Fig. 10 Flow index as a function of $d_{50}$ for all powders considered. 


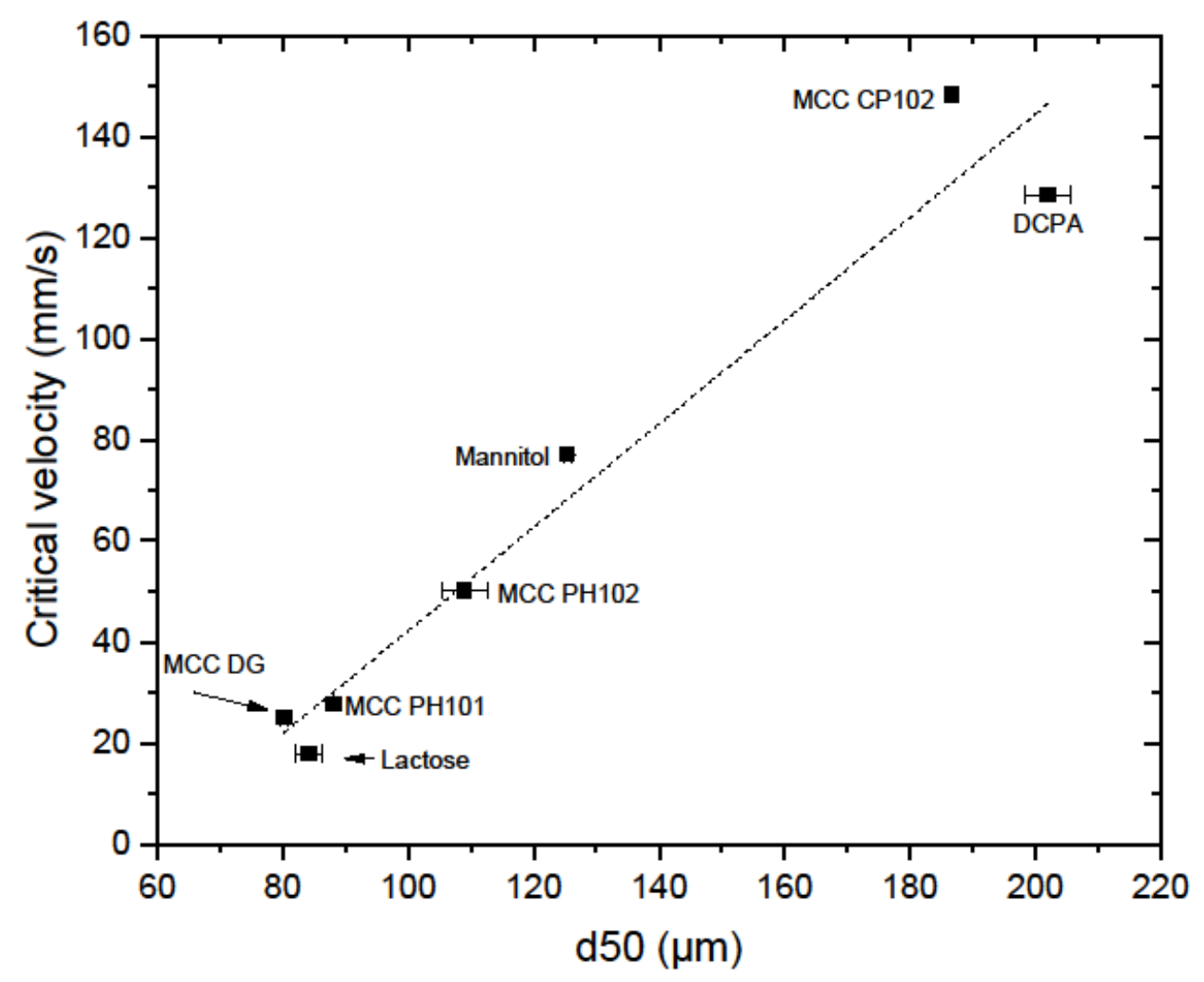

Fig. 11 Critical filling velocity as a function of the average particle size $d_{50}$. 


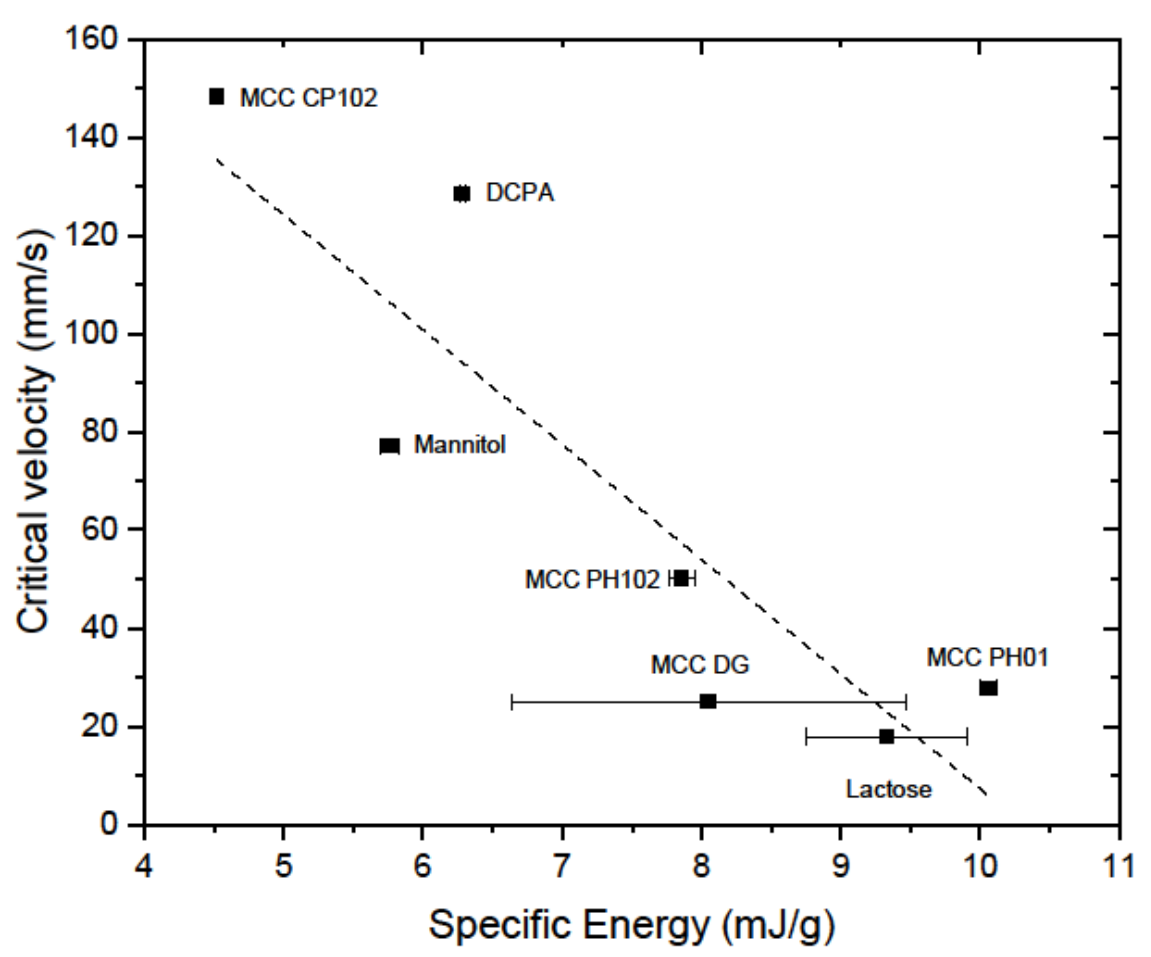

Fig. 12 Critical filling velocity as a function of specific energy. 


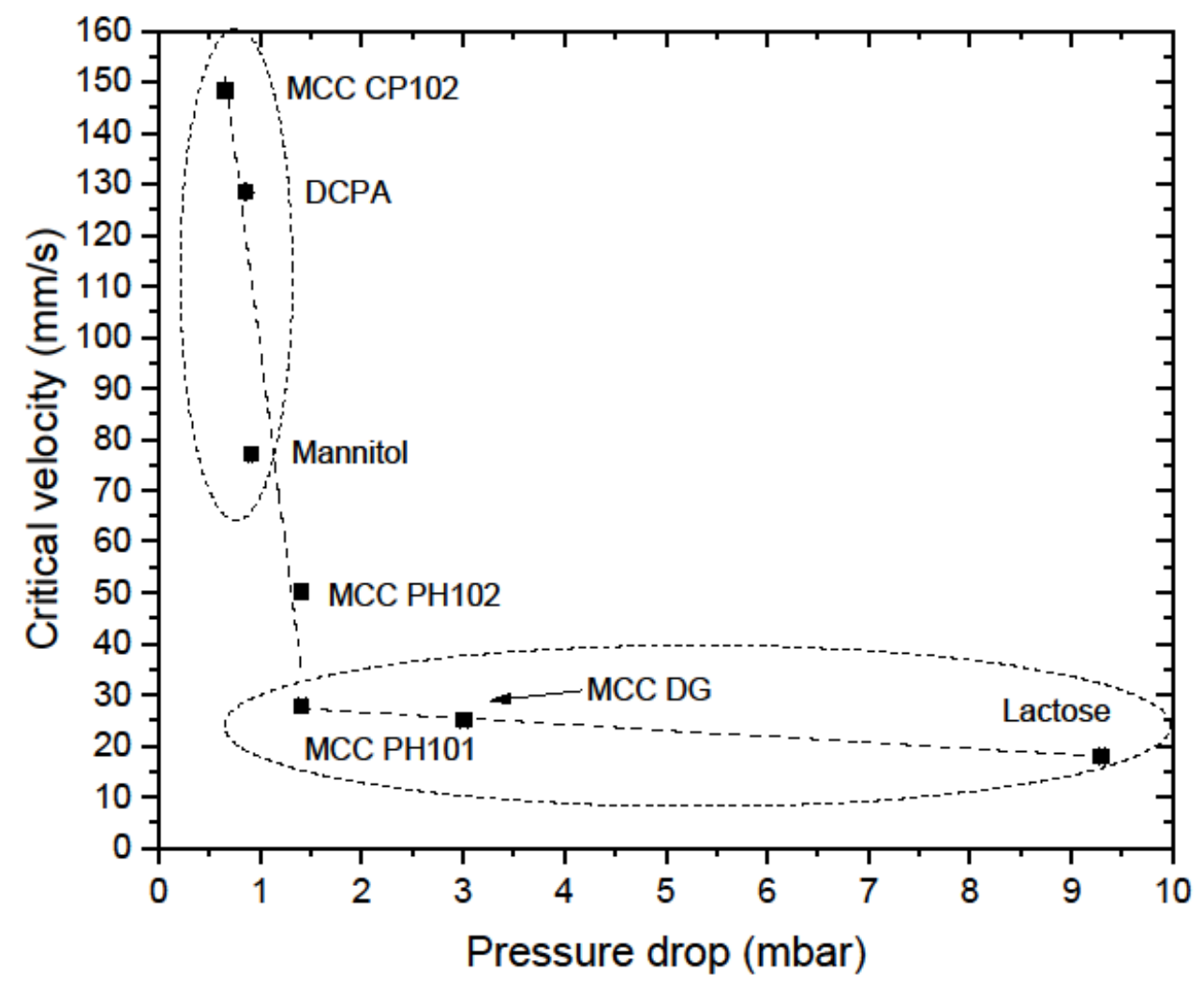

Fig. 13 Critical filling velocity as a function of pressure drop at $15 \mathrm{kPa}$ normal stress. 


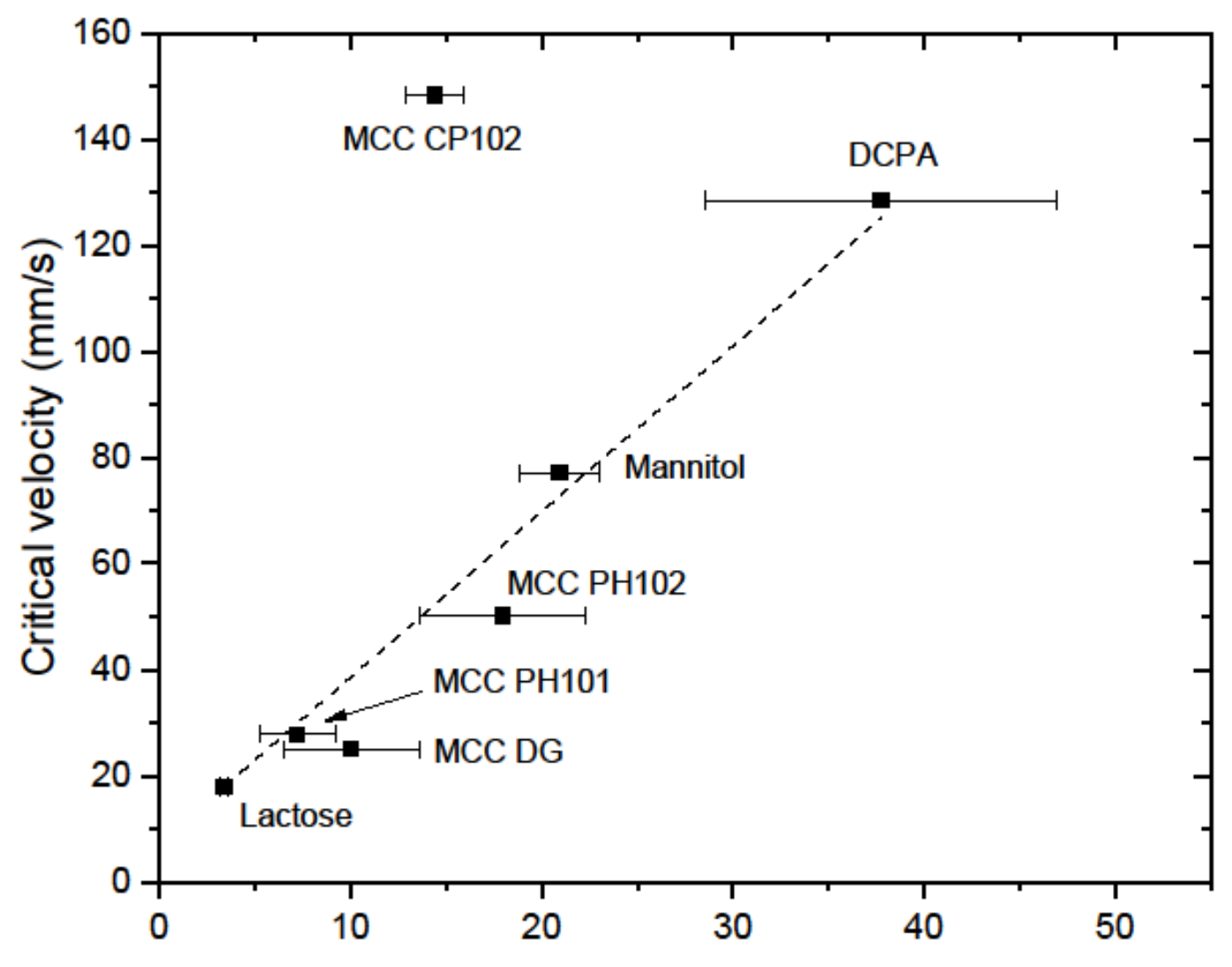

Flow function at $3 \mathrm{kPa}$

Fig. 14 Critical filling velocity as a function of flow function assessed at $3 \mathrm{kPa}$ consolidation stress. 


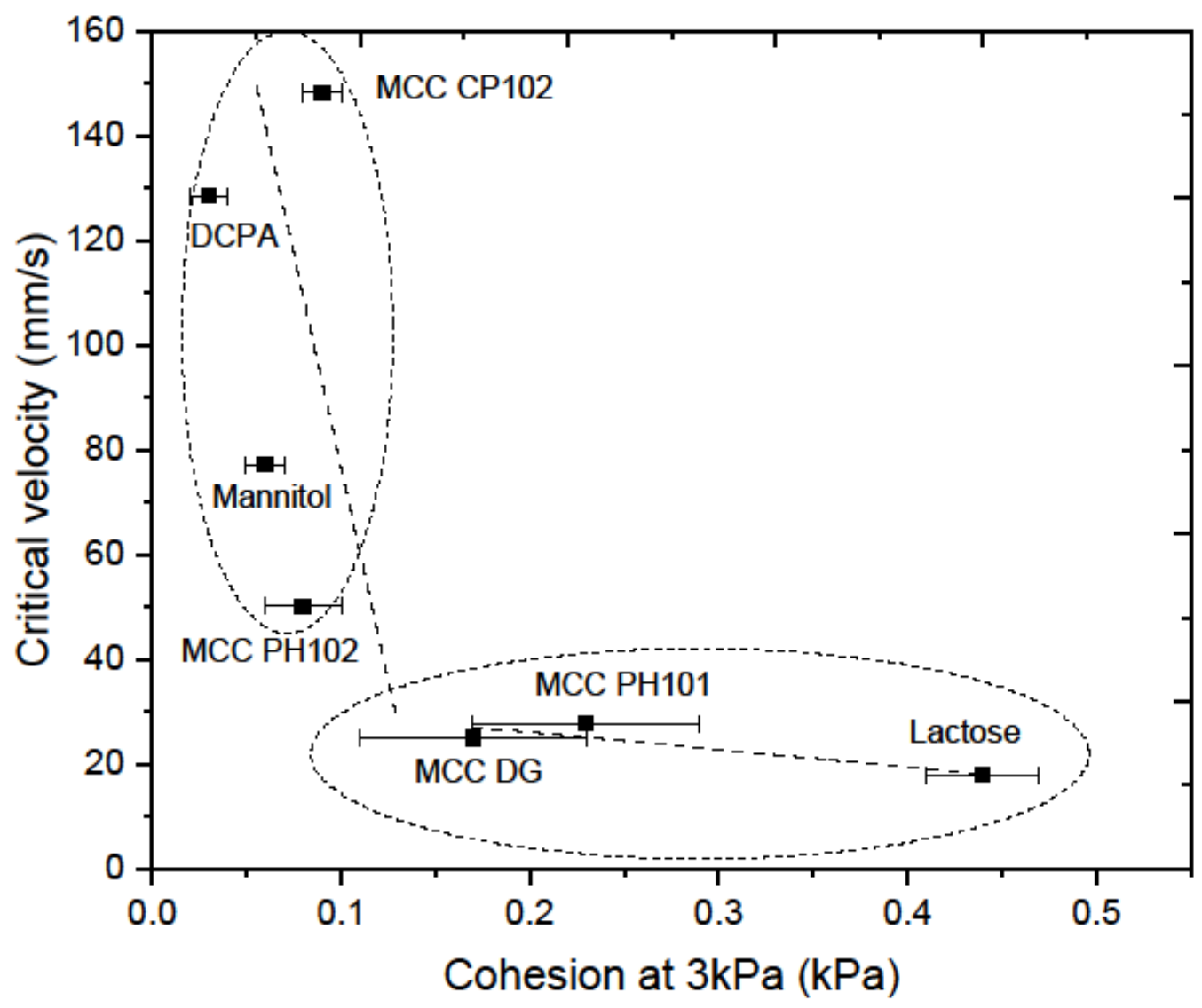

Fig. 15 Critical filling velocity as a function of cohesion assessed at $3 \mathrm{kPa}$ consolidation stress. 


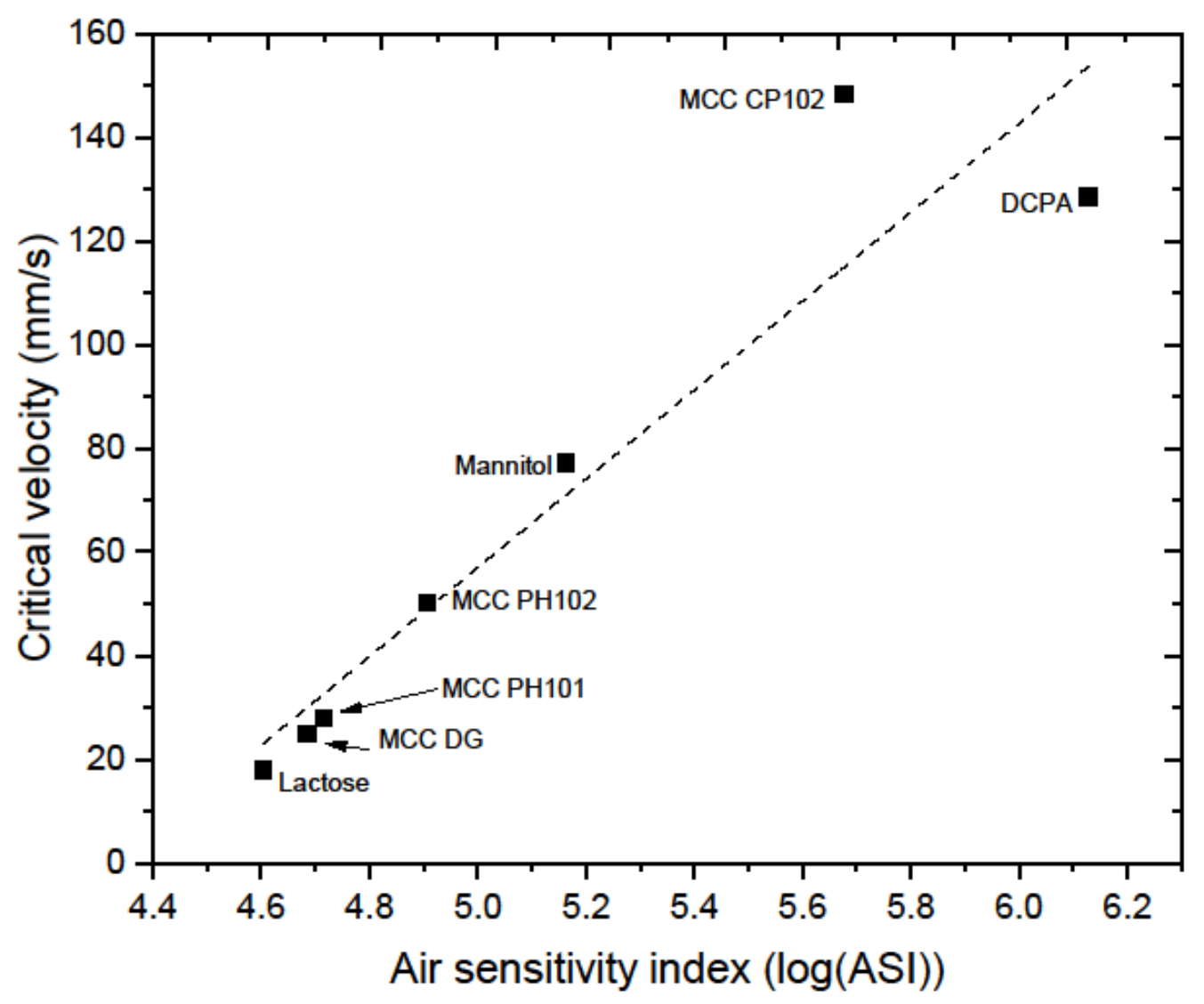

Fig. 16 Critical filling velocity as a function of the logarithm of air sensitivity for all powders considered. 